\title{
ALMA REDSHIFTS OF MILLIMETER-SELECTED GALAXIES FROM THE SPT SURVEY: THE REDSHIFT DISTRIBUTION OF DUSTY STAR-FORMING GALAXIES
}

\author{
A. Weiß ${ }^{1}$, C. De Breuck ${ }^{2}$, D. P. Marrone ${ }^{3}$, J. D. Vieira ${ }^{4}$, J. E. Aguirre ${ }^{5}$, K. A. Aird ${ }^{6}$, M. Aravena ${ }^{2}$, M. L. N. Ashby ${ }^{7}$, \\ M. Bayliss ${ }^{7,8}$, B. A. Benson ${ }^{9,10}$, M. Béthermin ${ }^{11}$, A. D. BigGs ${ }^{2}$, L. E. Bleem ${ }^{9,12}$, J. J. Bock ${ }^{4,13}$, M. BothWElL ${ }^{3}$, \\ C. M. Bradford ${ }^{13}$, M. Brodwin ${ }^{14}$, J. E. Carlstrom ${ }^{9,10,12,15,16}$, C. L. Chang ${ }^{9,10,16}$, S. C. ChapMaN ${ }^{17,18}$, T. M. CraWford ${ }^{9,15}$, \\ A. T. Crites ${ }^{9,15}$, T. De HaAn ${ }^{19}$, M. A. Dobbs ${ }^{19}$, T. P. Downes ${ }^{4}$, C. D. Fassnacht ${ }^{20}$, E. M. George ${ }^{21}$, M. D. Gladders $^{9,15}$, \\ A. H. Gonzalez ${ }^{22}$, T. R. Greve ${ }^{23}$, N. W. Halverson ${ }^{24}$, Y. D. Hezaveh ${ }^{19}$, F. W. High ${ }^{9,15}$, G. P. Holder ${ }^{19}$, W. L. Holzapfel ${ }^{21}$,

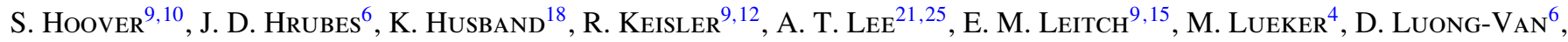 \\ M. Malkan ${ }^{26}$, V. McIntyre ${ }^{27}$, J. J. McMahon ${ }^{9,10,28}$, J. Mehl $^{9,15}$, K. M. Menten ${ }^{1}$, S. S. Meyer ${ }^{9,10,12,15}$, E. J. Murphy ${ }^{29}$,

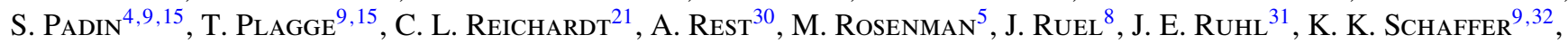 \\ E. Shirokoff ${ }^{4}$, J. S. Spilker ${ }^{3}$, B. Stalder ${ }^{7}$, Z. Staniszewski ${ }^{4,31}$, A. A. Stark ${ }^{7}$, K. Story ${ }^{9,12}$, K. VAnderlinde ${ }^{19}$, \\ N. WELIKALA ${ }^{33}$, AND R. WILLIAMSON ${ }^{9,15}$ \\ ${ }^{1}$ Max-Planck-Institut für Radioastronomie, Auf dem Hügel 69, D-53121 Bonn, Germany \\ 2 European Southern Observatory, Karl-Schwarzschild Straße, D-85748 Garching bei München, Germany \\ ${ }^{3}$ Steward Observatory, University of Arizona, 933 North Cherry Avenue, Tucson, AZ 85721, USA \\ ${ }^{4}$ California Institute of Technology, 1200 E. California Blvd., Pasadena, CA 91125, USA \\ ${ }^{5}$ University of Pennsylvania, 209 South 33rd Street, Philadelphia, PA 19104, USA \\ ${ }^{6}$ University of Chicago, 5640 South Ellis Avenue, Chicago, IL 60637, USA \\ ${ }^{7}$ Harvard-Smithsonian Center for Astrophysics, 60 Garden Street, Cambridge, MA 02138, USA \\ ${ }^{8}$ Department of Physics, Harvard University, 17 Oxford Street, Cambridge, MA 02138, USA \\ ${ }^{9}$ Kavli Institute for Cosmological Physics, University of Chicago, 5640 South Ellis Avenue, Chicago, IL 60637, USA \\ ${ }^{10}$ Enrico Fermi Institute, University of Chicago, 5640 South Ellis Avenue, Chicago, IL 60637, USA \\ ${ }^{11}$ Laboratoire AIM-Paris-Saclay, CEA/DSM/Irfu - CNRS - Université Paris Diderot, CEA-Saclay, Orme des Merisiers, F-91191 Gif-sur-Yvette, France \\ ${ }^{12}$ Department of Physics, University of Chicago, 5640 South Ellis Avenue, Chicago, IL 60637, USA \\ 13 Jet Propulsion Laboratory, 4800 Oak Grove Drive, Pasadena, CA 91109, USA \\ ${ }^{14}$ Department of Physics and Astronomy, University of Missouri, 5110 Rockhill Road, Kansas City, MO 64110, USA \\ ${ }^{15}$ Department of Astronomy and Astrophysics, University of Chicago, 5640 South Ellis Avenue, Chicago, IL 60637, USA \\ ${ }^{16}$ Argonne National Laboratory, 9700 S. Cass Avenue, Argonne, IL 60439, USA \\ ${ }^{17}$ Department of Physics and Atmospheric Science, Dalhousie University, Halifax, NS B3H 3J5 Canada \\ ${ }_{18}$ Institute of Astronomy, University of Cambridge, Madingley Road, Cambridge CB3 OHA, UK \\ ${ }^{19}$ Department of Physics, McGill University, 3600 Rue University, Montreal, Quebec H3A 2T8, Canada \\ ${ }^{20}$ Department of Physics, University of California, One Shields Avenue, Davis, CA 95616, USA \\ ${ }^{21}$ Department of Physics, University of California, Berkeley, CA 94720, USA \\ 22 Department of Astronomy, University of Florida, Gainesville, FL 32611, USA \\ ${ }^{23}$ Department of Physics and Astronomy, University College London, Gower Street, London WC1E 6BT, UK \\ ${ }^{24}$ Department of Astrophysical and Planetary Sciences and Department of Physics, University of Colorado, Boulder, CO 80309, USA \\ ${ }_{25}$ Physics Division, Lawrence Berkeley National Laboratory, Berkeley, CA 94720, USA \\ ${ }^{26}$ Department of Physics and Astronomy, University of California, Los Angeles, CA 90095-1547, USA \\ ${ }^{27}$ Australia Telescope National Facility, CSIRO, Epping, NSW 1710, Australia \\ ${ }^{28}$ Department of Physics, University of Michigan, 450 Church Street, Ann Arbor, MI, 48109, USA \\ ${ }^{29}$ Observatories of the Carnegie Institution for Science, 813 Santa Barbara Street, Pasadena, CA 91101, USA \\ ${ }^{30}$ Space Telescope Science Institute, 3700 San Martin Dr., Baltimore, MD 21218, USA \\ ${ }^{31}$ Physics Department, Center for Education and Research in Cosmology and Astrophysics, Case Western Reserve University, Cleveland, OH 44106, USA \\ ${ }^{32}$ Liberal Arts Department, School of the Art Institute of Chicago, 112 S Michigan Ave, Chicago, IL 60603, USA \\ ${ }^{33}$ Institut d'Astrophysique Spatiale, Bâtiment 121, Université Paris-Sud XI \& CNRS, F-91405 Orsay Cedex, France \\ Received 2012 October 31; accepted 2013 February 15; published 2013 March 28
}

\begin{abstract}
Using the Atacama Large Millimeter/submillimeter Array, we have conducted a blind redshift survey in the $3 \mathrm{~mm}$ atmospheric transmission window for 26 strongly lensed dusty star-forming galaxies (DSFGs) selected with the South Pole Telescope. The sources were selected to have $S_{1.4 \mathrm{~mm}}>20 \mathrm{mJy}$ and a dust-like spectrum and, to remove low- $z$ sources, not have bright radio $\left(S_{843 \mathrm{MHz}}<6 \mathrm{mJy}\right)$ or far-infrared counterparts $\left(S_{100 \mu \mathrm{m}}<1 \mathrm{Jy}\right.$, $S_{60 \mu \mathrm{m}}<200 \mathrm{mJy}$ ). We robustly detect 44 line features in our survey, which we identify as redshifted emission lines of ${ }^{12} \mathrm{CO},{ }^{13} \mathrm{CO}, \mathrm{C}$,, $\mathrm{H}_{2} \mathrm{O}$, and $\mathrm{H}_{2} \mathrm{O}^{+}$. We find one or more spectral features in 23 sources yielding a $\sim 90 \%$ detection rate for this survey; in 12 of these sources we detect multiple lines, while in 11 sources we detect only a single line. For the sources with only one detected line, we break the redshift degeneracy with additional spectroscopic observations if available, or infer the most likely line identification based on photometric data. This yields secure redshifts for $\sim 70 \%$ of the sample. The three sources with no lines detected are tentatively placed in the redshift desert between $1.7<z<2.0$. The resulting mean redshift of our sample is $\bar{z}=3.5$. This finding is in contrast to the redshift distribution of radio-identified DSFGs, which have a significantly lower mean redshift of $\bar{z}=2.3$ and for which only $10 \%-15 \%$ of the population is expected to be at $z>3$. We discuss the effect of gravitational
\end{abstract}


lensing on the redshift distribution and compare our measured redshift distribution to that of models in the literature.

Key words: cosmology: observations - early universe - galaxies: evolution - galaxies: high-redshift - ISM: molecules

Online-only material: color figures

\section{INTRODUCTION}

In the last decade, impressive progress has been made in our understanding of galaxy formation and evolution based on multi-wavelength deep field studies. Millimeter ( $\mathrm{mm})$ and submillimeter (submm) continuum observations demonstrated that luminous, dusty galaxies were a thousand times more abundant in the early universe than they are at present day (e.g., Smail et al. 1997; Blain et al. 1999; Chapman et al. 2005). The first surveys of the redshift distribution of dusty star-forming galaxies (DSFGs) suggested that the DSFG population peaks at redshift $\sim 2$ (e.g., Chapman et al. 2003, 2005), coeval with the peak of black hole accretion and the peak of the star formation rate density as measured in the optical/UV (e.g., Hopkins \& Beacom 2006). These studies suggested that the bulk of star formation activity in the universe at $z=2-3$ could be taking place in DSFGs, hidden from the view of optical/UV observations due to the high dust obscuration (e.g., Hughes et al. 1998; Blain et al. 1999).

Optical surveys now allow estimates of the history of star formation (the "Madau-Lilly" plot; Madau et al. 1996; Lilly et al. 1996; Hopkins \& Beacom 2006) out to $z \sim 8$ (e.g., Bouwens et al. 2010, 2011), but have uncertain dust extinction corrections. Submm observations can provide a more complete picture of the amount of highly obscured star formation over a large range of look-back times. However, such studies have been hampered by the difficulty of obtaining robust redshifts for DSFGs. This difficulty increases strongly as a function of redshift, and mainly arises from the coarse spatial resolution $\left(\sim 20^{\prime \prime}\right)$ of single-dish submm observations and the dust-obscured nature of the sources, which often prohibits identification of counterparts at other wavelengths. The solution has been to obtain higher spatial resolution data, usually at radio and/or mid-infrared wavelengths, in which the most likely counterpart to the submm emission could be identified (e.g., Ivison et al. 2002; Ashby et al. 2006; Pope et al. 2006; Wardlow et al. 2011; Yun et al. 2012). The slope of the spectral energy distributions (SEDs) of galaxies in the radio or mid-infrared (MIR), however, is such that the $K$-correction is positive, and galaxies become more difficult to detect at high redshifts. By contrast, the steeply rising spectrum of dusty sources leads to a negative $K$-correction for DSFGs at submm wavelengths, resulting in fluxes roughly constant with redshift (Blain \& Longair 1993). Therefore, while DSFGs may be discoverable at submm wavelengths at almost any redshift, their emission may be hidden at other wavelengths. Indeed, in submm surveys typically $50 \%$ of DSFGs lack robust counterparts (e.g., Biggs et al. 2011) albeit the fraction depends on the depth of the radio/MIR observations. This mismatch in the wavelength sensitivity could bias the DSFG redshift distribution, particularly at $z>3$.

A more reliable and complete method to obtain secure multi-wavelength identifications of DSFGs is to follow the single-dish detections up with $\mathrm{mm}$ interferometry. Prior to Atacama Large Millimeter/submillimeter Array (ALMA) this method has proven to be time-intensive, requiring entire nights of time detect a single source; the first sample detected blindly in the continuum with mm interferometry was published by Younger et al. (2007). A larger sample was published recently by Smolcic et al. (2012), which included optical spectroscopic redshifts for roughly half the sample and photometric redshift estimates the remaining sources in the sample which suggested that the previous spectroscopically determined redshift distributions (e.g., Chapman et al. 2005) were biased low.

A more direct and unbiased way to derive redshifts of DSFGs is via observations of molecular emission lines at millimeter wavelengths which can be related unambiguously to the (sub)mm continuum source. This method has only become competitive recently with the increased bandwidth of $\mathrm{mm} /$ submm facilities. Its power to measure reliable redshifts has been demonstrated in the case of SMMJ14009+0252 and HDF850.1 (Weiß et al. 2009a; Walter et al. 2012), two of the first DSFGs detected by SCUBA, for which other methods failed to deliver redshifts for more than a decade. While $\mathrm{CO}$ redshift surveys of a representative sample of DSFGs will remain observing time expensive until the operation of full ALMA, CO line redshifts for strongly lensed systems can be obtained easily (e.g., Swinbank et al. 2010; Cox et al. 2011; Frayer et al. 2011).

In the past, studies of strongly lensed sources have been limited to a handful of targets due to their rareness and the lack of large scale $\mathrm{mm} /$ submm surveys. This has changed dramatically over the past few years with the advent of large area surveys from Herschel (specifically H-ATLAS and HerMES; Eales et al. 2010; Oliver et al. 2010) and the South Pole Telescope (SPT-SZ; Carlstrom et al. 2011). These surveys have detected hundreds of strongly lensed high-redshift DSFGs (Vieira et al. 2010; Negrello et al. 2010). First CO redshift measurements at $\mathrm{mm}$ (Lupu et al. 2012) and centimeter (Harris et al. 2012) wavebands of H-ATLAS sources suggested that the lensed DSFGs lie within the same redshift range as unlensed, radioidentified sources (Chapman et al. 2005). Although a large overlap between the SPT and Herschel populations is expected, SPT's longer selection wavelength of $1.4 \mathrm{~mm}$ predicts a broader redshift distribution than Herschel detected sources and indeed photometric redshifts of DSFGs discovered by the SPT confirm this expectation (Greve et al. 2012).

In this paper, we present the results from an ALMA CO redshift survey of a sample of 26 strongly lensed DSFGs selected from $1300 \mathrm{deg}^{2}$ of SPT-SZ survey data (Carlstrom et al. 2011). The depth of the SPT-SZ survey data, which is sufficient to detect $S_{1.4 \mathrm{~mm}} \sim 20 \mathrm{mJy}$ sources at $5 \sigma$, combined with the flat redshift selection function of DSFGs at this wavelength (e.g., Blain \& Longair 1993), has produced an optimal sample for $\mathrm{mm}$ molecular line redshift searches in strongly lensed DSFGs. In an accompanying paper, Vieira et al. (2013) show that these sources are virtually all strongly lensed, while Hezaveh et al. (2013) report the associated lens modeling procedure.

In Section 2, we describe the target selection and observations. The biases on the observed redshift distribution, resulting from the source selection and the effect of gravitational lensing are discussed in Section 4. Our results are summarized in Section 5. Throughout this paper we have adopted a flat WMAP7 


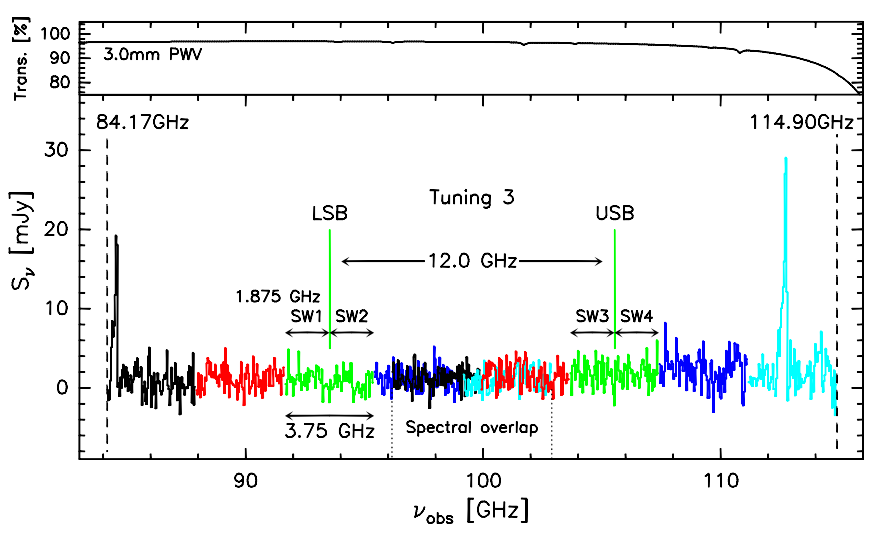

Figure 1. Spectral setup and frequency coverage of our five tunings (shown in different colors) in ALMA band 3 for the source SPT0103-45. In each tuning, four spectral windows covering $1.875 \mathrm{GHz}$ each were placed in contiguous pairs in the lower and upper sidebands (LSB/USB). Note that the frequency range 96.2-102.8 GHz (delimited by dotted vertical lines) is covered twice. The total spectral range is indicated by the dashed vertical lines. The top panel shows the atmospheric transmission across band 3 at Chajnantor for $3 \mathrm{~mm}$ precipitable water vapor (PWV).

cosmology, with $H_{0}=71 \mathrm{~km} \mathrm{~s}^{-1} \mathrm{Mpc}^{-1}, \Omega_{\Lambda}=0.73$ and $\Omega_{m}=0.27$ (Komatsu et al. 2011).

\section{OBSERVATIONS}

We observed a sample of 26 bright $\left(S_{1.4 \mathrm{~mm}}>20 \mathrm{mJy}\right)$, $1.4 \mathrm{~mm}$ selected SPT sources with ALMA. Sources were selected from the first $1300 \mathrm{deg}^{2}$ of the now complete $2500 \mathrm{deg}^{2}$ SPT-SZ survey (for more details on the survey, see Williamson et al. 2011; Story et al. 2012). The flux density cut is done on the initial raw flux density, and not the final de-boosted flux density, the details of which can be found in Vieira et al. (2010) and Crawford et al. (2010). To remove synchrotron dominated systems we required dust-like spectra between 1.4 and $2 \mathrm{~mm}\left(S_{1.4 \mathrm{~mm}} / S_{2.0 \mathrm{~mm}}>2\right.$; Vieira et al. 2010). In addition, we used far-infrared (FIR) and/or radio criteria to remove low-redshift contaminants (see Section 4.1). In order to refine the relatively coarse SPT source positions (the SPT's beam size is 1.05 at $1.4 \mathrm{~mm}$ ) we further required follow up observations at higher spatial resolution (typically $870 \mu \mathrm{m}$ images from the Large Apex BOlometer CAmera (LABOCA) or $1 \mathrm{~mm}$ data from the Submillimeter Array). Based on $1.4 \mathrm{~mm}$ flux densities, our Cycle 0 targets comprise a representative sample of the SPT sources meeting these selection criteria. This is shown in Appendix C where we present the SPT $1.4 \mathrm{~mm}$, LABOCA $870 \mu \mathrm{m}$, and Herschel-SPIRE $350 \mu \mathrm{m}$ flux density properties of this subsample compared to all SPT sources which have been observed with Herschel and LABOCA.

In order to optimize the ALMA observing efficiency, we assembled five groups of targets that lie within $15^{\circ}$ of each other on the sky-this restriction precluded a complete flux-limited sample. We excluded two sources with redshifts previously determined by Z-Spec (a wide-band, low resolution spectrometer operating between 190 and $310 \mathrm{GHz}$; see Bradford et al. 2004) on the Atacama Pathfinder Experiment (APEX) telescope and XSHOOTER (Vernet et al. 2011) or the FOcal Reducer and Spectrograph (FORS2; Appenzeller et al. 1998) on the ESO Very Large Telescope (VLT).

The ALMA observations were carried out in 2011 November and 2012 January in the Cycle 0 early science compact array configuration. We performed a spectral scan in the $3 \mathrm{~mm}$ atmospheric transmission window with five tunings in dual po-

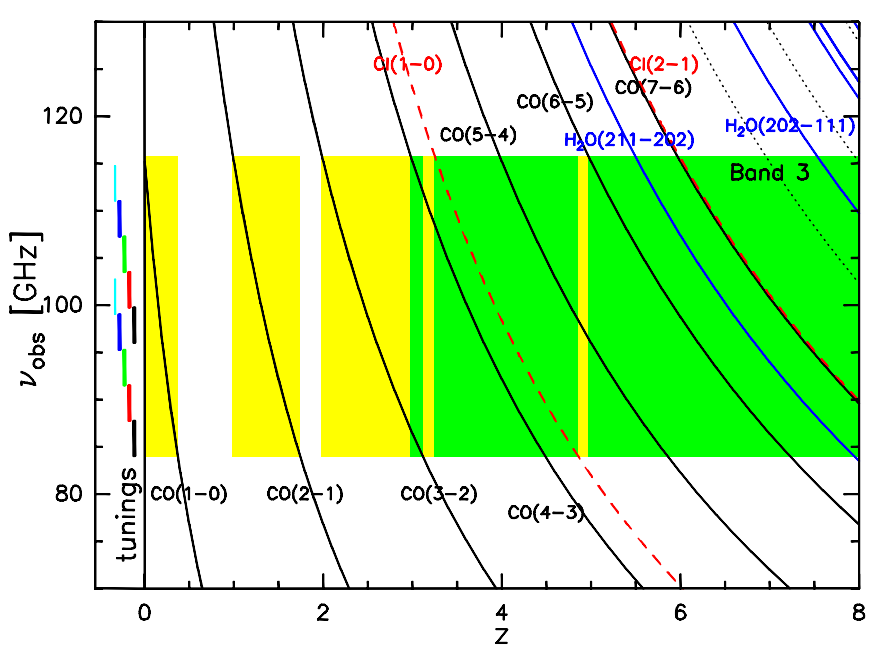

Figure 2. Spectral coverage of the $\mathrm{CO},[\mathrm{C} \mathrm{I}]$, and $\mathrm{H}_{2} \mathrm{O}$ emission lines as a function of redshift. The green shaded region marks the redshifts where two or more strong lines provide an unambiguous redshift, while the yellow region marks redshift range where only a single line is detectable. The five frequency tunings are shown in the left panel (see also Figure 1).

larization mode. Each tuning covers $7.5 \mathrm{GHz}$ in two $3.75 \mathrm{GHz}$ wide sidebands, each of which is covered by two $1.875 \mathrm{GHz}$ spectral windows in the ALMA correlator. This setup spans 84.2-114.9 GHz (with 96.2-102.8 GHz covered twice; see Figure 1), nearly the entire bandwidth of the Band 3 (84-116 GHz) receiver. Over this frequency range ALMA's primary beam is $61^{\prime \prime}-45^{\prime \prime}$. The observations employed between 14 and 17 antennas in different sessions, and resulted in typical synthesized beams of $7^{\prime \prime} \times 5^{\prime \prime}$ to $5^{\prime \prime} \times 3^{\prime \prime}($ FWHM) from the lowto high-frequency ends of the band. Each target was observed for $\sim 120 \mathrm{~s}$ in each tuning, or roughly 10 minutes per source in total, not including overheads.

Typical system temperatures for the observations were $T_{\text {sys }}=$ $60 \mathrm{~K}$. Flux calibration was performed on planets (Mars, Uranus, or Neptune) or Jupiter's moons (Callisto or Ganymede), with passband and phase calibration determined from nearby quasars. The data were processed using the Common Astronomy Software Application package (McMullin et al. 2007; Petry et al. 2012). Calibrated data cubes were constructed with a channel width of $19.5 \mathrm{MHz}\left(\sim 50-65 \mathrm{~km} \mathrm{~s}^{-1}\right.$ for the highest and lowest observing frequency). The typical noise per channel is $2 \mathrm{mJy}$ beam $^{-1}$ across the band and $1.4 \mathrm{mJy}$ beam $^{-1}$ between 96.0 and $102.8 \mathrm{GHz}$ where two tunings overlap. Continuum images generated from the full bandwidth have typical noise levels of $70 \mu \mathrm{Jy} \mathrm{beam}^{-1}$.

The spectral coverage of this experiment includes $\mathrm{CO}(1-0)$ for $0.003<z<0.36$ and one or more $\mathrm{CO}$ lines, between the (2-1) and (7-6) transitions, between $1.0<z<8.6$, with the exception of a small redshift "desert" between $1.74<z<2.00$ (see Figure 2). An additional redshift desert at $0.36<z<1.0$ is also present, but our high $1.4 \mathrm{~mm}$ flux density threshold effectively requires that our sources be gravitationally lensed (Section 4.1) and it is highly unlikely that sources at this redshift will be lensed (Section 4.2).

\section{RESULTS}

We detect $3 \mathrm{~mm}$ continuum emission with a high signalto-noise ratio ( $\mathrm{S} / \mathrm{N} 8-30)$ in all 26 sources; all sources remain spatially unresolved in these compact configuration data. Within the primary beam of ALMA we do not detect any other source 
Table 1

ALMA Source Positions

\begin{tabular}{|c|c|c|c|}
\hline \multirow[t]{2}{*}{ Short Name } & \multirow[t]{2}{*}{ Source } & R.A. & Decl. \\
\hline & & \multicolumn{2}{|c|}{$\mathrm{J} 2000$} \\
\hline SPT0103-45 & SPT-S J010312-4538.8 & $01: 03: 11.50$ & $-45: 38: 53.9$ \\
\hline SPT0113-46 & SPT-S J011308-4617.7 & 01:13:09.01 & $-46: 17: 56.3$ \\
\hline SPT0125-47 & SPT-S J012506-4723.7 & 01:25:07.08 & $-47: 23: 56.0$ \\
\hline SPT0125-50 & SPT-S J012549-5038.2 & $01: 25: 48.45$ & $-50: 38: 20.9$ \\
\hline SPT0128-51 & SPT-S J012809-5129.8 & $01: 28: 10.19$ & $-51: 29: 42.4$ \\
\hline SPT0243-49 & SPT-S J024307-4915.5 & $02: 43: 08.81$ & $-49: 15: 35.0$ \\
\hline SPT0300-46 & SPT-S J030003-4621.3 & 03:00:04.37 & $-46: 21: 24.3$ \\
\hline SPT0319-47 & SPT-S J031931-4724.6 & $03: 19: 31.88$ & $-47: 24: 33.7$ \\
\hline SPT0345-47 & SPT-S J034510-4725.6 & $03: 45: 10.77$ & $-47: 25: 39.5$ \\
\hline SPT0346-52 & SPT-S J034640-5204.9 & $03: 46: 41.13$ & $-52: 05: 02.1$ \\
\hline SPT0418-47 & SPT-S J041839-4751.8 & $04: 18: 39.67$ & $-47: 51: 52.7$ \\
\hline SPT0441-46 & SPT-S J044143-4605.3 & $04: 41: 44.08$ & $-46: 05: 25.5$ \\
\hline SPT0452-50 & SPT-S J045247-5018.6 & $04: 52: 45.83$ & $-50: 18: 42.2$ \\
\hline SPT0457-49 & SPT-S J045719-4932.0 & $04: 57: 17.52$ & $-49: 31: 51.3$ \\
\hline SPT0459-58 & SPT-S J045859-5805.1 & $04: 58: 59.80$ & $-58: 05: 14.0$ \\
\hline SPT0459-59 & SPT-S J045912-5942.4 & $04: 59: 12.34$ & $-59: 42: 20.2$ \\
\hline SPT0512-59 & SPT-S J051258-5935.6 & $05: 12: 57.98$ & $-59: 35: 41.9$ \\
\hline SPT0529-54 & SPT-S J052902-5436.5 & 05:29:03.09 & $-54: 36: 40.0$ \\
\hline SPT0532-50 & SPT-S J053250-5047.1 & $05: 32: 51.04$ & $-50: 47: 07.5$ \\
\hline SPT0550-53 & SPT-S J055001-5356.5 & $05: 50: 00.56$ & $-53: 56: 41.7$ \\
\hline SPT0551-50 & SPT-S J055138-5058.0 & $05: 51: 39.42$ & $-50: 58: 02.1$ \\
\hline SPT2103-60 & SPT-S J210328-6032.6 & $21: 03: 30.90$ & $-60: 32: 40.3$ \\
\hline SPT2132-58 & SPT-S J213242-5802.9 & $21: 32: 43.23$ & $-58: 02: 46.2$ \\
\hline SPT2134-50 & SPT-S J213404-5013.2 & $21: 34: 03.34$ & $-50: 13: 25.1$ \\
\hline SPT2146-55 & SPT-S J214654-5507.8 & $21: 46: 54.02$ & $-55: 07: 54.3$ \\
\hline SPT2147-50 & SPT-S J214720-5035.9 & $21: 47: 19.05$ & $-50: 35: 54.0$ \\
\hline
\end{tabular}

Notes. Source names are based on positions measured with the SPT. Source positions are based on the ALMA $3 \mathrm{~mm}$ continuum data.

at the sensitivity limit of our observations. Table 1 lists the ALMA $3 \mathrm{~mm}$ continuum positions, while the $3 \mathrm{~mm}$ continuum flux densities are given in Appendix $\mathrm{C}$ together with other photometric measurements.

Figure 3 presents the spectra. In total, we detect 44 line features with line integrated $\mathrm{S} / \mathrm{N}>5$ in our survey, which we identify as emission lines of ${ }^{12} \mathrm{CO},{ }^{13} \mathrm{CO}, \mathrm{C}$, $\mathrm{H}_{2} \mathrm{O}$, and $\mathrm{H}_{2} \mathrm{O}^{+}$. Our spectra can be grouped into three categories:

1. Spectra with no line features (three sources).

2. Spectra with a single line feature (11 sources). For these spectra we cannot determine the redshift unambiguously and use other spectroscopic and photometric measurements to constrain the redshift.

3. Spectra with multiple line features (12 sources). In this case, a unique redshift solution can be derived from the ALMA $3 \mathrm{~mm}$ spectral scans alone.

Table 2 summarizes the detected line features and the derived redshifts. Uncertainties for the redshifts are based on Gaussian fits to the line profiles. The identification of the ambiguous features is discussed in Section 3.2.

\subsection{Additional Spectroscopic Observations}

For five of the sources in our sample for which we have detected only a single line in our $3 \mathrm{~mm}$ scan, we determine the redshift using additional $\mathrm{mm} /$ submm or optical spectroscopy. We describe the observations and show the spectra in Appendix A.

\subsection{Ambiguous Cases}

The most likely candidates for a single line feature in the $3 \mathrm{~mm}$ band are redshifted transitions of $\mathrm{CO}$ up to $J=3-2$ (see
Figure 2). The $\mathrm{CO}(4-3)$ and $\mathrm{CO}(5-4)$ lines may also appear as single lines across the band in cases where the $\mathrm{CI}\left({ }^{3} P_{1} \rightarrow{ }^{3} P_{0}\right)$ line falls out of the covered frequency range or may be too faint to be detected (the lowest flux density ratio between $\mathrm{CI}\left({ }^{3} P_{1} \rightarrow{ }^{3} P_{0}\right)$ and $\mathrm{CO}(4-3)$ or $\mathrm{CO}(5-4)$ that we observe in our survey is $<0.15(3 \sigma))$. Single-line spectra cannot result from CO transitions of $J=6-5$ or higher or molecular lines that can appear at flux densities comparable to $\mathrm{CO}$ (such as $\mathrm{H}_{2} \mathrm{O}$; van der Werf et al. 2010, 2011), because these lines would be accompanied by another line within the observing band (see Figure 2). The detection of FIR fine structure lines, such as $122 \mu \mathrm{m}$ and $205 \mu \mathrm{m}$ [N II] and $158 \mu \mathrm{m}$ [C II] would require extreme redshifts $(z>11)$ which are inconsistent with $\mathrm{mm} /$ submm continuum measurements.

Photometric measurements allow us to discriminate between the possible line assignments in our single-line sources. The thermal dust emission of our sources is sampled by $3 \mathrm{~mm}$ ALMA, $2 \& 1.4 \mathrm{~mm}$ SPT, and $870 \mu \mathrm{m} \mathrm{LABOCA}$ as well as 500, 350, and $250 \mu \mathrm{m}$ Herschel-SPIRE observations. The photometry is given in Appendix C.

For the fitting of the thermal dust continuum we have used the method described in Greve et al. (2012) which uses a graybody fit with a spectral slope of $\beta=2$ and an optically thin/thick transition wavelength of $100 \mu \mathrm{m}$, where the only free parameters are the dust luminosity and the dust temperature, $T_{\text {dust }}$. As in Greve et al. (2012), we exclude data points shortward of $\lambda_{\text {rest }}=50 \mu \mathrm{m}$ from the fit because a single-temperature SED model typically cannot match both sides of the SED peak simultaneously due to the presence of dust at multiple temperatures. Both the spectral slope and transition wavelength affect the derived dust temperatures. For the present purpose, we seek only a consistent measure of the location of the SED peak in each source; the "temperatures" should not be interpreted as physical temperatures. The dust temperature is better derived using the source structural information that will be available with lens models based on high spatial resolution ALMA observations (Hezaveh et al. 2013), which will help constrain the dust opacity (e.g., Weiß et al. 2007).

Given the fundamental degeneracy between $T_{\text {dust }}$ and redshift due to Wien's displacement law, it is not possible to solve for $z$ and $T_{\text {dust }}$ simultaneously. We therefore determine $T_{\text {dust }}$ for each of the possible redshifts and compare these to the dust temperature distribution for targets with unambiguous redshifts (see Table 2), including the two SPT sources with previously known redshifts from Greve et al. (2012) which share the same selection criteria than the sample discussed here. For these sources we find a mean of $T_{\text {dust }}=37.2 \pm$ 8.2 $\mathrm{K}$ and no apparent trend with redshift (see Figure 4, left). Based on the distribution of the temperatures in this sub-sample (19 sources) we have calculated the probability for each of the 4 dust temperature/redshift options for the 6 sources with a single detected line and ambiguous redshifts. This analysis strongly prefers a single redshift for one additional source (SPT0452-50; see Appendix B). We have included SPT0452-50 in the sample of sources with known redshifts, bringing the total to 20 .

Figure 4 shows the five remaining sources with ambiguous redshifts. We retain only dust temperatures with probabilities $>10 \%$, and find two plausible line identifications/redshifts for four sources. In one case this threshold only rules out $\mathrm{CO}(2-1)$, leaving three plausible redshifts. Table 2 lists the possible redshifts together with the implied dust temperatures. Entries in bold face show the most likely redshift solution. 

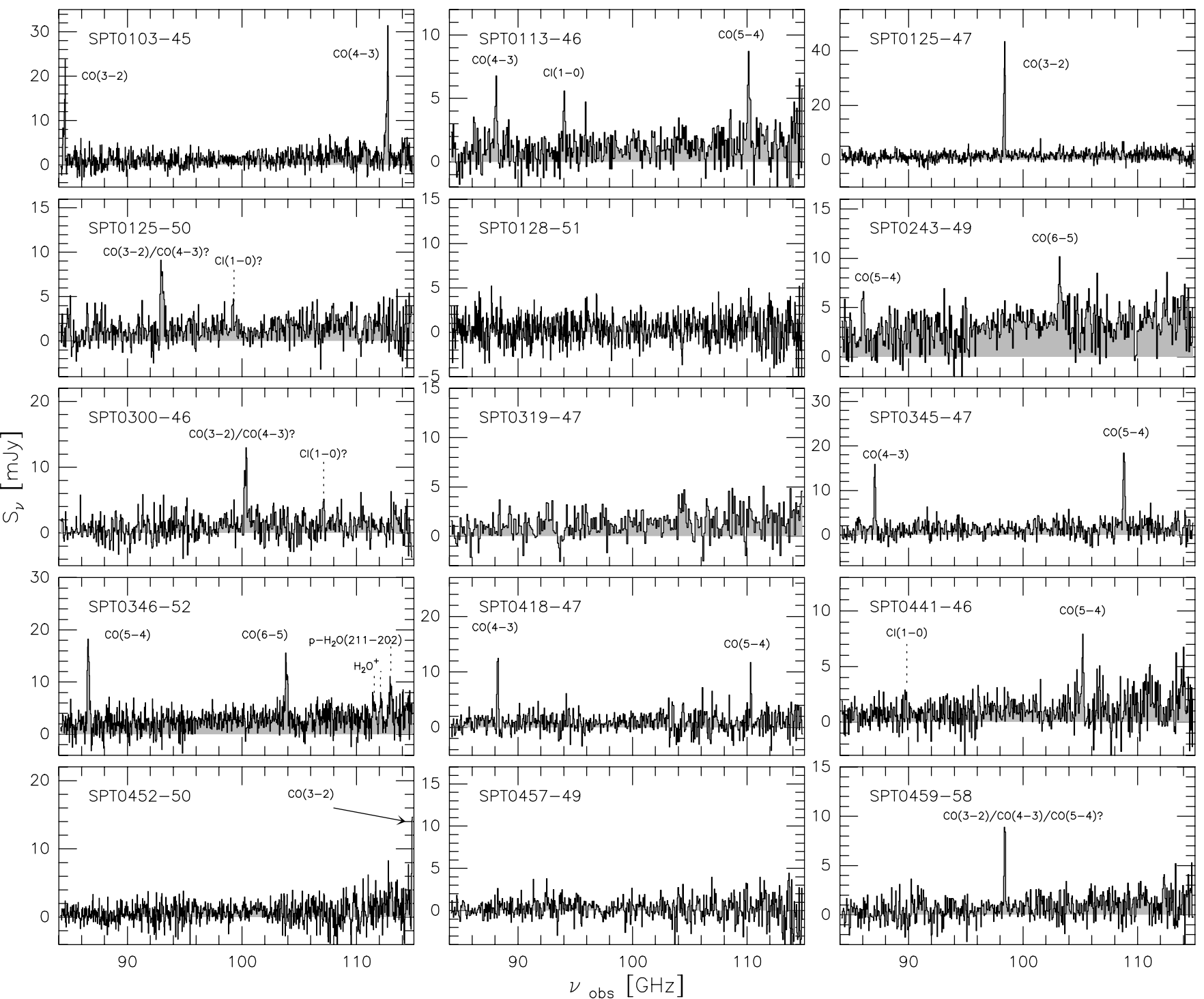

Figure 3. Spectra for target galaxies in the ALMA the $3 \mathrm{~mm}$ band. Spectra are shown at a resolution of $40-70 \mathrm{MHz}\left(\sim 100-250 \mathrm{~km} \mathrm{~s}^{-1}\right)$ depending on the line width and the signal-to-noise ratio.

In the case of the three sources without line features, we derive photometric redshifts based on the FIR data using the mean dust temperature of the objects with unambiguous redshifts. This places these three sources between $z=3.3-4.2$ (see right column of Table 2). Of the two redshift ranges for which we cannot observe a CO line, the $0.36<z<1$ range can be excluded because the SED would then imply $T_{\text {dust }}$ lower than the dust temperatures of the Milky Way and other spiral galaxies ( $T_{\text {dust }}<15 \mathrm{~K}$ for all sources) and due to the small lensing probability (Section 4.2). The galaxies may then be in the redshift desert at $z=1.74-2.00$ or at higher redshift with CO line intensities below our detection threshold. Our redshift survey sensitivity was intended to detect $\mathrm{CO}$ lines out to $z \sim 6$, based on molecular gas estimates from the dust continuum, and strong detections of emission lines in $90 \%$ of the targets out to $z=5.7$ lends credibility to the sensitivity target. However, two of our non-detections are among the $1.4 \mathrm{~mm}$-faintest sources which leaves open the possibility that the line sensitivity is inadequate in these cases, albeit we do detect $\mathrm{CO}$ lines at similar $1.4 \mathrm{~mm}$ flux density in the survey. Yet, estimates of the CO (and CI) line intensities based on the dust continuum observations alone require several strong assumptions (e.g., on the gas-to-dust mass ratio and the molecular gas excitation). Thus we cannot rule out that these systems represent a class of galaxies with lower than expected line to continuum ratio, with the lines falling below our detection limit. If we place these three galaxies at $z=$ $1.74-2.00$, we obtain low ( $T_{\text {dust }} \approx 20 \mathrm{~K}$ ), but still plausible dust temperatures given the $T_{\text {dust }}$ distribution in our sub-sample with known redshifts. We note that this redshift identification is by no means secure, but represents the lowest plausible redshift range given the estimates based on the photometric data discussed above.

A discussion of the nine individual cases which have zero or one CO line detected with ALMA and no additional spectroscopic observations is presented in Appendix C.

\section{DISCUSSION}

\subsection{Redshift Biases due to Source Selection Criteria}

From our line identifications in Table 2, it is apparent that the lowest secure redshift detected in our survey is at $z=$ 2.010. Only five sources are possibly at $z \leqslant 2$ (assuming that 


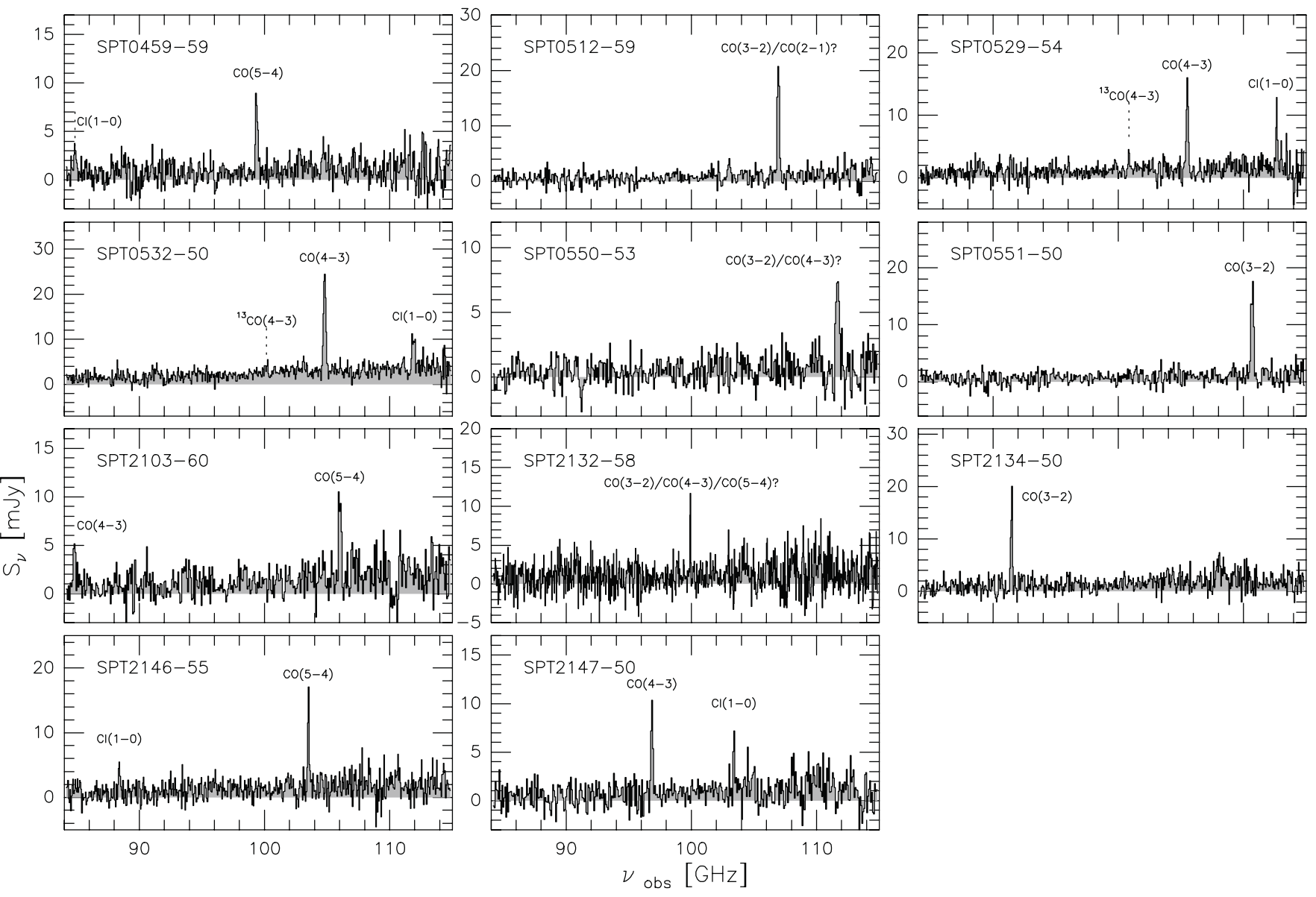

Figure 3. (Continued)
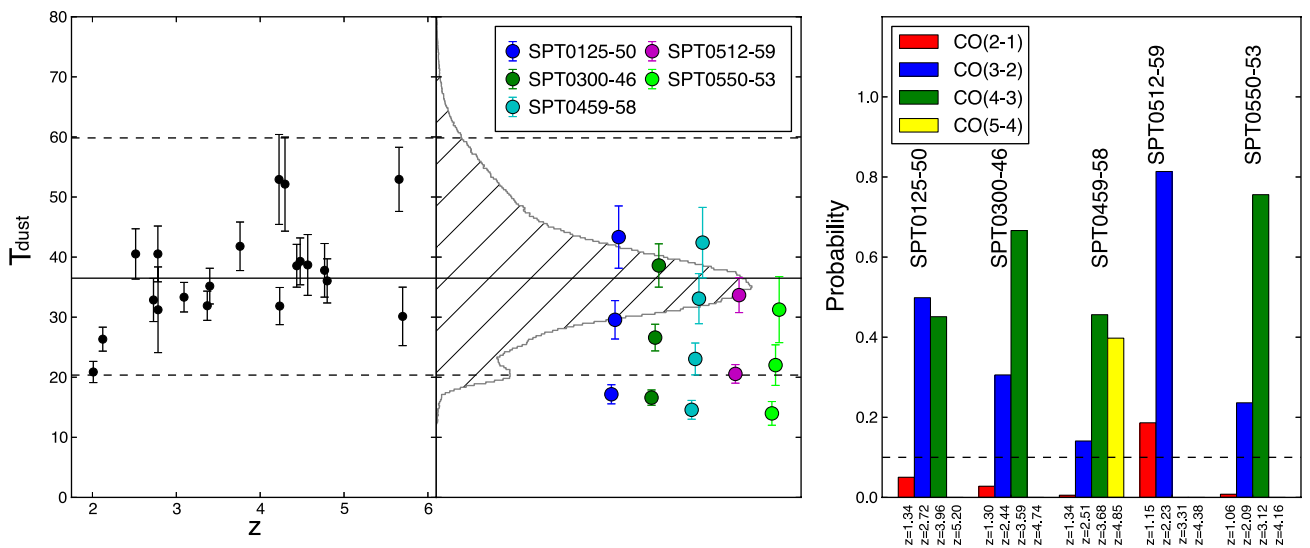

Figure 4. Left: dust temperatures for the sources in our sample with unambiguous redshifts. Center: combined histogram of dust temperatures derived from the posterior likelihood distributions for the sources with unambiguous redshifts. Overplotted are the dust temperatures determined for each redshift option for those sources with uncertain redshift; horizontal spacing is arbitrary. The solid and dashed lines show the median and $95 \%$ confidence interval dust temperatures for those sources with unambiguous redshifts. Right: probability for the single line detected in our ALMA spectrum to be identified as one of the four possible CO transitions for the five sources with ambiguous redshifts. The probabilities were calculated by comparing the dust temperature associated with each line identification to the dust temperature distribution of our sources with known redshifts. The horizontal dashed line shows a probability of $10 \%$, the cut off above which we consider the line identification to be plausible.

sources without a line detection fall into the redshift desert $z=1.74-2.00)$. This is in contrast to the expectation from radioidentified DSFG redshift surveys, where typically $\sim 50 \%$ of all sources fall into the redshift range $0.5<z<2.0$ (e.g., Chapman et al. 2005; Wardlow et al. 2011).

Part of this discrepancy arises from our source selection criteria. In order to select strongly lensed, dusty high-redshift sources from the SPT $1.4 \mathrm{~mm}$ maps efficiently, additional criteria are used to distinguish the high- $z$ population from the low $z$ and synchrotron-dominated sources that dominate the number counts of $S_{1.4 \mathrm{~mm}}>20 \mathrm{mJy}$ sources. Vieira et al. (2010) present a discussion of the classification of these populations and the details on how to distinguish them. Below, we provide a summary of the selection criteria and discuss their impact.

We first select sources whose mm flux is dominated by thermal dust emission. This step is based on the ratio of 
Table 2

Redshifts and Line Identification

\begin{tabular}{|c|c|c|c|c|}
\hline Source & $z$ & $\begin{array}{c}T_{\text {dust }} \\
(\mathrm{K})\end{array}$ & Lines & Comment \\
\hline \multicolumn{5}{|c|}{ Secure Redshifts } \\
\hline SPT0103-45 & $3.0917(3)^{\mathrm{a}}$ & $33.3 \pm 2.5$ & $\mathrm{CO}(3-2) \& \mathrm{CO}(4-3)$ & \\
\hline SPT0113-46 & $4.2328(5)$ & $31.8 \pm 3.1$ & $\mathrm{CO}(4-3), \mathrm{CI}(1-0) \& \mathrm{CO}(5-4)$ & \\
\hline SPT0125-47 & $2.51480(7)$ & $40.7 \pm 4.2$ & $\mathrm{CO}(3-2)$ & \multirow[t]{3}{*}{$\mathrm{CO}(1-0)$ from the ATCA } \\
\hline SPT0243-49 & $5.699(1)$ & $30.1 \pm 4.9$ & $\mathrm{CO}(5-4) \& \mathrm{CO}(6-5)$ & \\
\hline SPT0345-47 & $4.2958(2)$ & $52.1 \pm 7.8$ & $\mathrm{CO}(4-3) \& \mathrm{CO}(5-4)$ & \\
\hline SPT0346-52 & $5.6559(4)$ & $52.9 \pm 5.3$ & $\mathrm{CO}(5-4), \mathrm{CO}(6-5), \mathrm{H}_{2} \mathrm{O} \& \mathrm{H}_{2} \mathrm{O}^{+}$ & \\
\hline SPT0418-47 & $4.2248(7)$ & $52.9 \pm 7.5$ & $\mathrm{CO}(4-3) \& \mathrm{CO}(5-4)$ & \\
\hline SPT0441-46 & $4.4771(6)$ & $39.3 \pm 3.9$ & $\mathrm{CI}(1-0) \& \mathrm{CO}(5-4)$ & $\mathrm{CI}(1-0)$ feature low $\mathrm{S} / \mathrm{N},[\mathrm{C}$ II $]$ confirmation with APEX \\
\hline SPT0452-50 & $2.0104(2)$ & $20.9 \pm 1.8$ & $\mathrm{CO}(3-2)$ & Alternative redshifts excluded due to lack of higher J CO lines \\
\hline SPT0459-59 & $4.7993(5)$ & $36.0 \pm 3.7$ & $\mathrm{CI}(1-0) \& \mathrm{CO}(5-4)$ & \\
\hline SPT0529-54 & $3.3689(1)$ & $31.9 \pm 2.4$ & $\mathrm{CO}(4-3), \mathrm{CI}(1-0) \&{ }^{13} \mathrm{CO}(4-3)$ & \\
\hline SPT0532-50 & $3.3988(1)$ & $35.1 \pm 3.0$ & $\mathrm{CO}(4-3), \mathrm{CI}(1-0) \&{ }^{13} \mathrm{CO}(4-3)$ & \\
\hline SPT0551-50 & $2.1232(2)$ & $26.3 \pm 2.0$ & $\mathrm{CO}(3-2)$ & \multirow[t]{2}{*}{ VLT C IV $1550 \AA$ detection } \\
\hline SPT2103-60 & $4.4357(6)$ & $38.6 \pm 3.5$ & $\mathrm{CO}(4-3) \& \mathrm{CO}(5-4)$ & \\
\hline SPT2132-58 & $4.7677(2)$ & $37.8 \pm 4.5$ & $\mathrm{CO}(5-4)$ & \multirow[t]{4}{*}[\mathrm{C}_{\mathrm{II}}]{ from APEX } \\
\hline SPT2134-50 & $2.7799(2)$ & $40.5 \pm 4.6$ & $\mathrm{CO}(3-2)$ & \\
\hline SPT2146-55 & $4.5672(2)$ & $38.7 \pm 5.1$ & $\mathrm{CI}(1-0) \& \mathrm{CO}(5-4)$ & \\
\hline SPT2147-50 & $3.7602(3)$ & $41.8 \pm 4.1$ & $\mathrm{CO}(4-3) \& \mathrm{CI}(1-0)$ & \\
\hline SPT0538-50 & 2.783 & $31.2 \pm 7.1$ & $\mathrm{CO}(7-6), \mathrm{CO}(8-7)$, Si IV $1400 \AA$ & ZSpec/VLT from Greve et al. (2012); no ALMA data \\
\hline SPT2332-53 & 2.738 & $32.9 \pm 3.6$ & $\mathrm{CO}(7-6), \mathrm{Ly} \alpha, \mathrm{C}$ IV $1549 \AA$ & ZSpec/VLT from Greve et al. (2012); no ALMA data \\
\hline \multicolumn{5}{|c|}{ Ambiguous redshifts } \\
\hline SPT0125-50 & $3.9592(5)$ & $43.3 \pm 5.2$ & $\mathrm{CO}(4-3) \& \mathrm{CI}(1-0)$ & $\mathrm{CI}(1-0)$ feature low $\mathrm{S} / \mathrm{N}$ \\
\hline$\ldots$ & $2.7174(6)$ & $29.5 \pm 3.2$ & $\mathrm{CO}(3-2)$ & Alternative ID if $\mathrm{CI}(1-0)$ is not real \\
\hline SPT0300-46 & $3.5956(3)$ & $38.6 \pm 3.6$ & $\mathrm{CO}(4-3) \& \mathrm{CI}(1-0)$ & $\mathrm{CI}(1-0)$ feature low $\mathrm{S} / \mathrm{N}$ \\
\hline$\cdots$ & $2.4474(3)$ & $26.7 \pm 2.2$ & $\mathrm{CO}(3-2)$ & Alternative ID if $\mathrm{CI}(1-0)$ is not real \\
\hline SPT0459-58 & $3.6854(2)$ & $32.0 \pm 4.5$ & $\mathrm{CO}(4-3)$ & \\
\hline$\cdots$ & $4.8565(2)$ & $40.8 \pm 6.0$ & $\mathrm{CO}(5-4)$ & Similarly likely ID \\
\hline$\ldots$ & $2.5142(1)$ & $22.4 \pm 2.9$ & $\mathrm{CO}(3-2)$ & \\
\hline SPT0512-59 & $2.2335(2)$ & $33.2 \pm 3.0$ & $\mathrm{CO}(3-2)$ & \\
\hline$\ldots$ & $1.1557(1)$ & $20.4 \pm 1.6$ & $\mathrm{CO}(2-1)$ & \\
\hline SPT0550-53 & $3.1286(5)$ & $30.6 \pm 4.6$ & $\mathrm{CO}(4-3)$ & \\
\hline$\cdots$ & $2.0966(4)$ & $21.6 \pm 2.9$ & $\mathrm{CO}(3-2)$ & \\
\hline \multicolumn{5}{|c|}{ No CO line detections } \\
\hline SPT0128-51 & $\cdots$ & $\cdots$ & No lines & $z=1.74-2.00 ? ; z_{\text {photo }}=3.8 \pm 0.5$ for $T_{\text {dust }}=37.2 \mathrm{~K}$ \\
\hline SPT0319-47 & $\cdots$ & $\cdots$ & No lines & $z=1.74-2.00 ? ; z_{\text {photo }}=4.2 \pm 0.2$ for $T_{\text {dust }}=37.2 \mathrm{~K}$ \\
\hline SPT0457-49 & $\cdots$ & $\cdots$ & No lines & $z=1.74-2.00 ? ; z_{\text {photo }}=3.3 \pm 0.2$ for $T_{\text {dust }}=37.2 \mathrm{~K}$ \\
\hline
\end{tabular}

Notes. In case of ambiguous redshifts, preferred solutions are shown in bold.

a The number in brackets is the redshift uncertainty in the last decimal derived from Gaussian fits to the line profiles.

1.4-2.0 mm flux density and is efficient at removing any synchrotron-dominated source from the sample, the majority of which are flat-spectrum radio quasars (FSRQs) and have previously been cataloged at radio wavelengths. We impose a flux density cut on the sample of dust-dominated sources of $S_{1.4 \mathrm{~mm}}>20 \mathrm{mJy}$ based on the raw fluxes determined on the $1.4 \mathrm{~mm}$ maps.

The second step is to use external FIR catalogs to remove ("veto") low-redshift sources from the sample of dusty sources. Any source detected in the IRAS Faint Source Catalog (IRAS-FSC; Moshir et al. 1992) at 60 or $100 \mu \mathrm{m}$ (which implies $S_{60} \mu \mathrm{m}<200 \mathrm{mJy}$ and $S_{100} \mu \mathrm{m}<1$ Jy over the entire SPT field) is omitted from our source sample. This removes $\sim 70 \%$ of the dusty sources from our sample. Every dusty source with a counterpart in both the SPT and IRAS-FSC catalogs has a published spectroscopic redshift at $z<0.03$ and is not strongly lensed.

The third step is to use external radio catalogs to remove low-redshift and radio-loud sources from the sample of dusty sources. Any source detected in the $843 \mathrm{MHz}$ Sydney University
Molonglo Sky Survey (SUMSS; Bock et al. 1998; with a $\sim 6$ mJy $5 \sigma$ flux density threshold over the entire SPT field) is omitted from our source sample. The SUMSS veto removes an additional $\sim 15 \%$ of the dusty sources which passed the IRAS veto. This step is intended to ensure that no FSRQs were allowed into the sample. The mean radio flux density reported in the SUMSS catalog for these sources is $\left\langle S_{843 \mathrm{MHz}}\right\rangle=52 \mathrm{mJy}$, well above the catalog threshold.

The effect of these selections on the redshift distribution of the $1.4 \mathrm{~mm}$ sources targeted in this study depends on the intrinsic radio-IR SEDs of the DSFGs. Figure 5 shows the redshift limits beyond which different radio-IR SEDs pass our source veto criteria. We show here well-studied examples of quiescent and star-forming local galaxies, as well as an example for a high-redshift, radio-loud active galactic nucleus (AGN) host galaxy. The figure demonstrates that galaxies which follow the local radio-FIR correlation and have relatively cold dust temperatures $\left(T_{\text {dust }} \lesssim 30 \mathrm{~K}\right.$, e.g., M51) would pass our source selection criteria at relatively low redshift $(z \gtrsim 0.5)$. 


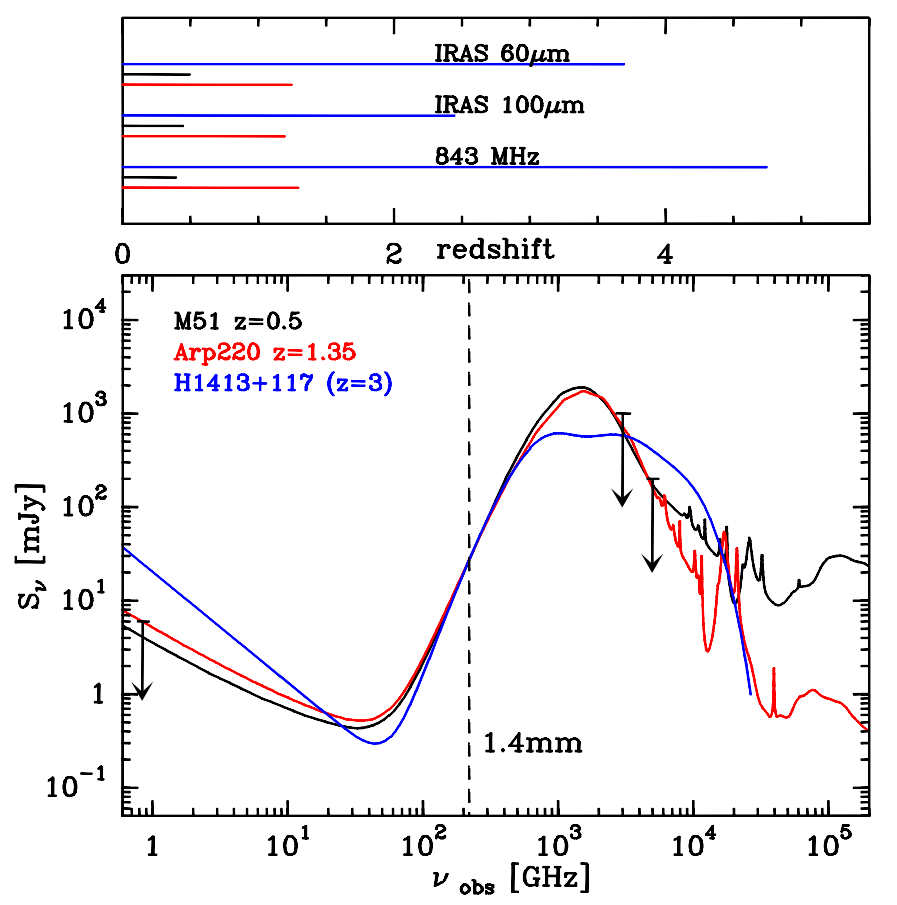

Figure 5. Top: redshift bias due to our IRAS 60 and $100 \mu \mathrm{m}$, and $843 \mathrm{MHz}$ radio flux vetos. The bars show the redshift range for which specific radio-to-IR SEDs are excluded from our sample. The color coding of the bars corresponds to galaxies shown in the bottom part of the figure. Bottom: radio to optical SEDs of M51 (the Whirlpool Galaxy), Arp 220 (the nearest ultraluminous infrared galaxy) and $\mathrm{H} 1413+117$ (the Cloverleaf QSO). These galaxies represent a range of possible SED types and are normalized to $S_{1.4 \mathrm{~mm}}=28 \mathrm{mJy}$ (the mean $1.4 \mathrm{~mm}$ flux density of our sample). The dashed horizontal line shows our selection wavelength of $1.4 \mathrm{~mm}$. The arrows show the $843 \mathrm{MHz}, 100 \mu \mathrm{m}$ and $60 \mu \mathrm{m}$ upper limits used for our source selection. The SEDs are shown for the lowest redshift (value indicated in the figure) for which each source matches our selection criteria, except for $\mathrm{H} 1413+117$ which is shown at $z=3.0$.

Sources with Arp-220-like SEDs would pass our selection criteria at higher redshifts $(z \gtrsim 1.4)$. Other local and high- $z$ IR luminous sources, including M82, SMM J2135-0102 ("The Eyelash"-Swinbank et al. 2010), and HR10 (Stern et al. 2006, not shown), are allowed at redshifts similar to Arp 220. Sources with FIR SEDs dominated by hotter dust (due to AGN heating, as in H1413+117, also known as "The Cloverleaf"; Benford et al. 1999) than is typical for star-forming systems would be found in IRAS and excluded from the sample out to $z \sim 3$.

The SUMSS veto may exclude a few source classes from our sample. Figure 5 shows that systems with much higher radio power than implied by the radio-IR correlation, such as lensed radio-loud AGN with significant dust emission (e.g., the Cloverleaf), are excluded from our sample over a large redshift range. This veto may also exclude lensed DSFGs at $z \lesssim 1.5$ (coincidentally close to the IRAS redshift veto limit), where the radio-FIR correlation predicts the radio emission will exceed the SUMSS limit. Finally, DSFGs lensed by foreground galaxies with radio-active AGN and residual FSRQs will be excluded in a redshift-unbiased way by this veto.

\subsection{Redshift Biases due to Gravitational Lensing}

The high $1.4 \mathrm{~mm}$ flux density cut of our target selection implies that even the most infrared-luminous galaxies are too faint to be included in the SPT dusty-source sample at $z \gtrsim 0.5$ without assistance from gravitational lensing $\left(L_{\mathrm{IR}}>3 \times\right.$ $10^{13} L_{\odot}$ for a Arp-220-like SED). This expectation is confirmed by our ALMA high-angular resolution imaging that resolves our

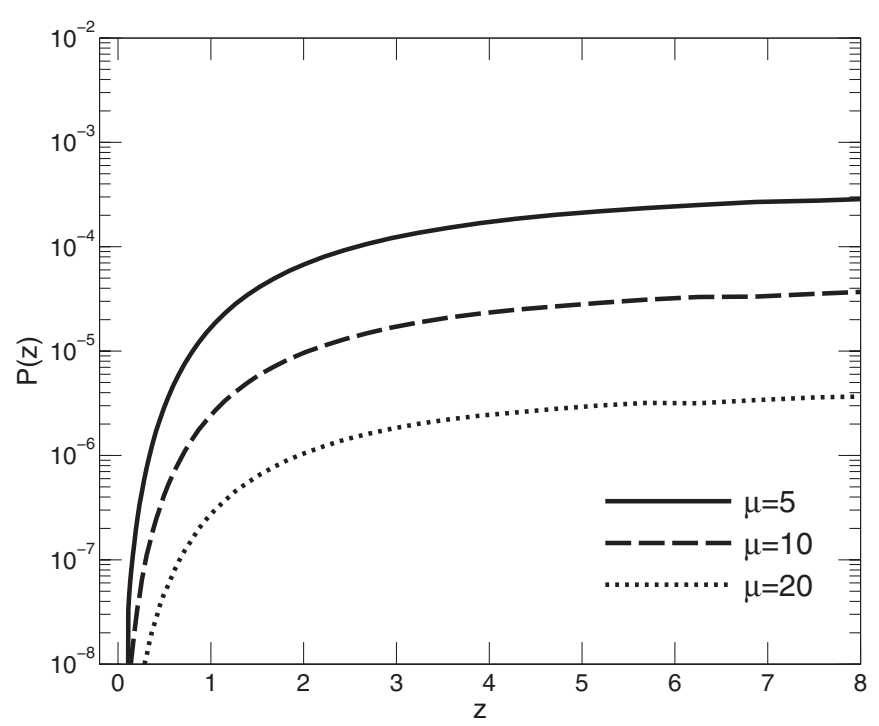

Figure 6. Probability of strong gravitational lensing as a function of redshift for different source magnifications $(\mu)$ calculated from the models of Hezaveh \& Holder (2011). The model assumes no size evolution for the underlying DSFG population. The figure demonstrates the strong decline of the lensing probability for $z \lesssim 1.5$, independent of the magnification.

sources into arcs or Einstein rings-hallmarks of gravitational lensing (Vieira et al. 2013). The redshift-dependent probability of strong gravitational lensing therefore has important effects on our redshift distribution. In Figure 6, we show the differential probability of strong lensing versus redshift, calculated from the models of Hezaveh \& Holder (2011) and Hezaveh et al. (2012), which use gravitational lensing by a realistic population of intervening halos to match the observed number counts of bright DSFGs. The strong evolution in the lensing probability (the fractional volume at each redshift subject to high magnification), a factor of 20 between $z \sim 2$ and $z \sim 0.5$, demonstrates that the requirement that we find lensed sources strongly suppresses sources at $z \lesssim 1.5$. For $z \gtrsim 2$ the lensing probability varies much more slowly, implying weaker effects on the lensed source counts.

At higher redshifts, other lensing effects can more significantly alter the normalized redshift distribution, $d n / d z$, especially changes in source sizes. To evaluate such effects, we compare an assumed intrinsic redshift distribution to the model distribution of strongly lensed sources $\left(S_{1.4 \mathrm{~mm}}>15 \mathrm{mJy}\right.$, consistent with the deboosted $1.4 \mathrm{~mm}$ flux densities of our sources, see Appendix C). As discussed in Hezaveh et al. (2012), the selection of a sample of millimeter-bright DSFGs, lensed by intervening galaxies, will preferentially identify those with more compact emission regions. This implies that the observed redshift distribution could be biased if DSFGs undergo a size evolution with redshift.

Observationally, it is well established that high-redshift DSFGs are significantly larger than local ULIRGs. In the high-redshift $(z \gtrsim 2)$ sources the star-forming regions extend over $\sim 5 \mathrm{kpc}$ diameter, while lower-redshift $(z \lesssim 1)$ ULIRGs typically form stars in kpc-sized regions (see, e.g., Tacconi et al. 2006; Engel et al. 2010 and references therein). Whether DSFGs undergo a size evolution in the redshift range $z=$ 1.5-6, the relevant redshift range for our study, is, however, largely unknown due to the small number of high-redshift objects for which spatially resolved observations of the submm emission region exist and the large diversity of morphologies. 
Evidence for extended molecular gas reservoirs $(>10 \mathrm{kpc} \mathrm{di}-$ ameter) has been found in some DSFGs out to redshift $z \approx 4$ (e.g., Genzel et al. 2003; Ivison et al. 2010, 2011; Younger et al. 2010; Carilli et al. 2011; Riechers et al. 2011a) while the molecular gas distribution in IR luminous AGN host galaxies, which have been measured out to redshift $z=6.4$, are typically more compact ( $\sim 2-3 \mathrm{kpc}$ diameter, e.g., Walter et al. 2004, 2009). These differences, however, mainly reflect the diversity of submm-detected objects and possibly an evolutionary link between DSFGs and AGN host galaxies (Riechers et al. 2011b) rather than an overall size evolution of submm-selected high- $z$ galaxies.

In Figure 7, we compare different size-evolution scenarios, where the intrinsic distribution was prescribed to be consistent with the observed redshift distribution from radio-identified DSFGs including recent spectroscopic data from the literature (Chapman et al. 2005; Capak et al. 2008; Coppin et al. 2009; Daddi et al. 2009a, 2009b; Riechers et al. 2010; Banerji et al. 2011; Walter et al. 2012). The figure demonstrates that the effect of gravitational lensing on the observed redshift distribution is relatively small when there is no size evolution or increasing source sizes with redshift. For example, in the redshift range $z=2-4$ the difference between $d n / d z$ derived from the unlensed and lensed sources is smaller than $\sim 20 \%$ in both cases. In the case of no size evolution the observed redshifted distribution is displaced by $\Delta z \sim 0.3$ toward higher redshifts compared to the unlensed case. Given the steep increase of $d n / d z$ between $z=1-2$ of the redshift distribution (Chapman et al. 2005; Banerji et al. 2011), this shift causes an underestimate of the source counts in this redshift interval by roughly a factor of two which may explain the low number of $z<2$ objects detected in our survey. For decreasing source sizes with redshift (as suggested by optical observations; Fathi et al. 2012) the difference between the observed and intrinsic redshift distribution can become significant also for $z>3$, with the counts of the high-redshift galaxies increased compared to the intrinsic distribution.

A compilation of the effective source radii for $z=1-6$ derived from an analysis of the dust SEDs of unlensed submm detected DSFGs and quasi-stellar object (QSO) host galaxies has been published in Greve et al. (2012). Their Figure 5 shows the submm source radii as a function of redshift. The size of the highest redshift sources $(z=5-6)$ in this diagram tend to fall below the average size of $z=1-3$ objects, but as mentioned above, these high-redshift sources are all QSO host galaxies and as such cannot be taken as evidence for a size evolution of the whole DSFG population. The sample of source radii in the literature (Tacconi et al. 2008; Engel et al. 2010; Rujopakarn et al. 2011), which were directly measured from high-resolution imaging, show no clear evidence for size evolution above $z>0.4$. In the absence of conclusive observational constraints, it is difficult to quantify the redshift bias due to gravitational lensing. We note, however, that making our observed redshift distribution consistent with an intrinsic distribution like the one from Chapman et al. (2005) would require an extreme growth of DSFGs between $z=6$ and $z=2(r=0.2 \mathrm{kpc}$ to $2.5 \mathrm{kpc}$ in $2.3 \mathrm{Gyr}$, see Figure 7). Likewise, a modest evolution $(r=1.5 \mathrm{kpc}$ at $z=6-2.5 \mathrm{kpc}$ at $z=2$, using the QSO size measurements as lower limits to the size of DSFGs at $z=6$, see above) results in a steeper redshift distribution than that implied by our most likely redshifts. Both suggest that gravitational lensing is unlikely to be the dominant source for the differences in $d n / d z$ between the present sample and the radio-identified samples.

\subsection{The Redshift Distribution}

Even with the conservative choice of taking all ambiguous sources to be at their lowest redshift option (see Table 2), at least $50 \%$ of the SPT sample is at $z>3$. Only five sources are possibly at $z \leqslant 2$ (assuming that sources without a line detection fall into the redshift desert $z=1.74-2.00$ ), consistent with the expectations for a sample of strongly lensed objects. Our sample mean redshift is $\bar{z}=3.5$. This redshift distribution is in contrast to that of radio identified DSFGs which have a significantly lower mean redshift of $\bar{z}=2.3$ and for which only $10 \%-15 \%$ of the population is expected to be at $z>3$ (e.g., Chapman et al. 2005; Wardlow et al. 2011).

A potential difference between our redshift distribution and the $850-\mu \mathrm{m}$-selected samples in the literature arises from the interaction of the SED of the typical DSFG and the selection wavelength. This has been discussed in several papers, including Greve et al. (2008) and Smolcic et al. (2012). It has been argued that $850 \mu \mathrm{m}$ selection results in lower redshift samples than $1.4 \mathrm{~mm}$ selection because the negative $K$-correction ceases once the SED peak is redshifted into the detection band, which occurs at lower redshift for shorter wavelength observations. Because our sources have been selected at $1.4 \mathrm{~mm}$ (SPT) and also observed at $870 \mu \mathrm{m}$ (LABOCA), we can examine the effect that $850 \mu \mathrm{m}$ selection would have on our sample. The flux ratio as a function of redshift is shown in Figure 8, and it reveals a modest decrease of the $870 \mu \mathrm{m} / 1.4 \mathrm{~mm}$ flux ratio for increasing redshift. Our observations therefore support the notion that $850 \mu \mathrm{m}$ selection will preferentially remove sources at the highest redshifts. We caution, however, that this effect will operate only on the fainter population of high-redshift sources, those near to the detection limit where the $850 \mu \mathrm{m}$ may fall below the detection threshold while the $1.4 \mathrm{~mm}$ signal remains detectable.

Some studies of submm selected galaxies from blank field surveys presented evidence for a correlation between observed submm flux density and the source redshift (e.g., Ivison et al. 2002, 2007; Pope et al. 2005; Biggs et al. 2011). If confirmed, this could imply a possible bias toward higher redshift for our study if the intrinsic IR luminosity of our sample is on average higher than that of unlensed $\mathrm{mm} /$ submm selected samples. So far, lens models based on spatially resolved images of the $870 \mu \mathrm{m}$ continuum are only available for four SPT sources (Hezaveh et al. 2013). These have magnifications of $\mu=5-21$ with a mean of $\bar{\mu}=14$. The gravitational flux amplification of the SPT sources has also been discussed in Greve et al. (2012). They derive $\bar{\mu}=11-22$ based on an analysis of the FIR properties of 11 SPT sources compared to unlensed samples, in reasonable agreement with the lens models. Adopting an average magnification of $\bar{\mu}=15$ for the sources studied here, our sample is expected to cover intrinsic flux densities of $S_{1.4 \mathrm{~mm}}=1.0-3.0 \mathrm{mJy}$ and $S_{870 \mu \mathrm{m}}=1.7-9.5 \mathrm{mJy}$ with means of $\bar{S}_{1.4 \mathrm{~mm}}=1.8 \mathrm{mJy}$ and $\bar{S}_{870 \mu \mathrm{m}}=5.4 \mathrm{mJy}$. These intensities ranges are well matched with unlensed source flux densities observed in mm/submm blank fields surveys (e.g., Borys et al. 2003; Coppin et al. 2006; Pope et al. 2006; Austermann et al. 2009; Weiß et al. 2009b), which implies that our sample should be representative for the submm selected galaxy population at $z>1.5$. We further note that the claimed correlation between observed submm flux density and source redshift has recently been questioned (Wardlow et al. 2011; Karim et al. 2013).

An additional difference between this sample and earlier spectroscopic measurements of the DSFG redshift distribution is the radio selection. As noted above, previous DSFG redshift 


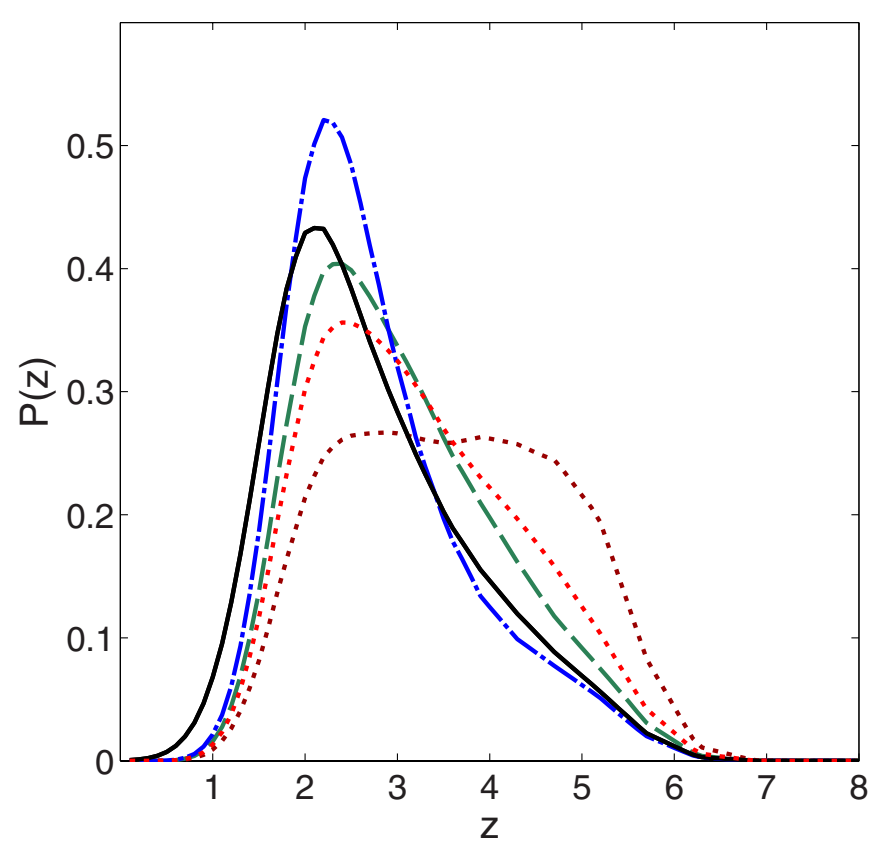

Figure 7. Comparison between an assumed intrinsic redshift distribution $(d n / d z$, solid black) consistent with spectroscopic observations (see text for references) and distributions modified by gravitational lensing (using the models described in Hezaveh \& Holder 2011) under different size evolution scenarios. The green dashed line shows the bias to the redshift distribution due to gravitational lensing assuming no size evolution versus redshift. The blue dot-dashed line show the bias to the redshift distribution due to gravitational lensing if the size of DSFGs increases with redshift, from $r=1 \mathrm{kpc}$ at $z=2$ to $3 \mathrm{kpc}$ at $z=5$. The red dotted line shows the bias of the redshift distribution due to gravitational lensing if the size of DSFGs decreases moderately with redshift ( $r=2.5 \mathrm{kpc}$ at $z=2$ as measured for DSFGs (Engel et al. 2010), to $r=1.5 \mathrm{kpc}$ at $z=6$ using the measured submm QSO host sizes (Walter et al. 2009) as lower bound to the size of DSFGs). The maroon dotted line exemplifies the extreme size evolution which would be required to bring the redshift distribution of Chapman et al. (2005) into agreement with our observations $(r=2.5 \mathrm{kpc}$ at $z=2$ to $r=0.2 \mathrm{kpc}$ at $z=6)$.

searches have primarily relied upon radio counterpart identification to provide optical spectroscopy targets and therefore have a radio detection requirement. Here we have excluded sources with bright radio counterparts, which might be expected to oppositely bias the sample. However, a comparison of the submmradio flux density ratio distribution for the radio-identified sample of Chapman et al. (2005) and the similar ratio (corrected for differences in observing frequency) constructed from SUMSS and SPT measurements for our SUMSS-vetoed sources shows that these objects emit a much larger fraction of their energy in the radio than even the most extreme sources in Chapman et al. (2005; see their Figure 7). Likewise, sources that pass our SUMSS radio-veto are not biased toward larger submm-radio flux density ratios than radio selected samples from the literature due to the shallowness of the SUMSS survey. Therefore this veto should not preferentially exclude low-redshift DSFGs, though optical spectroscopic measurements of the excluded sources will be useful in determining which source classes and which redshifts dominate the excluded objects.

The determination of the shape of our redshift distribution is currently hampered by the eight ambiguous redshifts. In Figure 9 (left) we compare two redshift distributions, one using the lowest redshift option for all sources, and the other assuming the most likely redshift. In the first case, our redshift distribution shows some evidence for a peak at $z \approx 3$, consistent with the findings of radio identified DSFGs, and then decreases out to $z \sim 6$. The

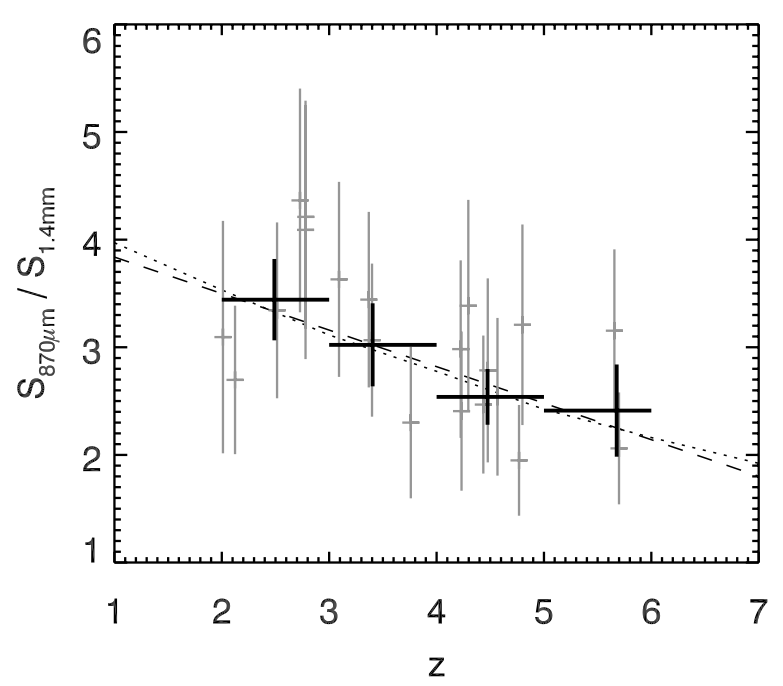

Figure 8. Observed $870 \mu \mathrm{m}$ to $1.4 \mathrm{~mm}$ flux density ratio as a function of redshift for our sample of 20 sources with unambiguous spectroscopic redshifts. The gray points show the individual measurements and their error bars taking absolute calibration uncertainties into account. The black crosses show the mean flux density ratio in redshift bins of $\Delta z=1$ centered at the weighted mean $z$. The dashed line is a linear fit to the data $S_{870} \mu \mathrm{m} / S_{1.4 \mathrm{~mm}}=4.18-0.34 \mathrm{z}$ for $z=2-6)$. The dotted line shows the expectation for a Arp-220-like dust SED.

Table 3

Measured Redshift Distribution for SPT Sources

\begin{tabular}{lccc}
\hline \hline$z$ & $N^{\mathrm{a}}$ & $d n / d z$ & \pm \\
\hline $1.5-2.5$ & 6 & 0.21 & 0.09 \\
$2.5-3.5$ & 8 & 0.29 & 0.10 \\
$3.5-4.5$ & 9 & 0.32 & 0.11 \\
$4.5-5.5$ & 3 & 0.11 & 0.06 \\
$5.5-6.5$ & 2 & 0.07 & 0.05
\end{tabular}

Notes. Reported redshifts are the most probable redshifts for 28 sources, 20 of which have unambiguous spectroscopic redshifts (see Section 3.2)

a Number of sources per bin as listed in Table 2 including two SPT sources with previously known redshifts from Greve et al. (2012).

decrease, however, is much shallower than suggested from radio identified DSFGs. In the latter case our redshift distribution rises up to $z \approx 4$ and falls off at higher redshift. Within the errors both distributions are consistent with a flat redshift distribution between $z=2-4$. Note that to these distributions we have added two additional strongly lensed SPT sources from Greve et al. (2012).

We adopt the redshift distribution informed by our dust temperatures and other data ("SPT best" in Figure 9) for the discussion which follows, and report the values for $d n / d z$ in Table 3.

Figure 9 (center) highlights the large difference between our results and previous redshift surveys. Compared to previous surveys with spectroscopic redshifts that rely on radio counterpart identification (Chapman et al. 2005; Capak et al. 2008; Coppin et al. 2009; Daddi et al. 2009a, 2009b; Riechers et al. 2010; Banerji et al. 2011; Walter et al. 2012) we find a far greater fraction of high-redshift sources. As discussed earlier, gravitational lensing may explain part of this discrepancy if DSFGs are smaller at high redshifts, though extreme evolution is required to explain the full difference. Recent work based on $\mathrm{CO}(1-0)$-derived redshifts for a DSFG sample selected from the H-ATLAS survey (Harris et al. 2012, not shown here) implies a redshift distribution in agreement with Chapman et al. (2005). 

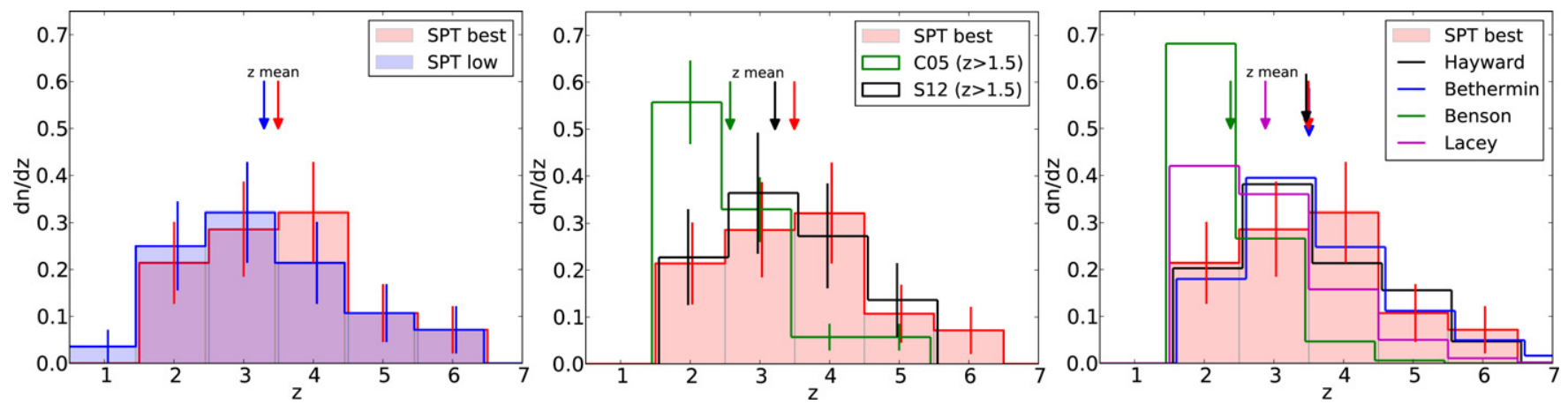

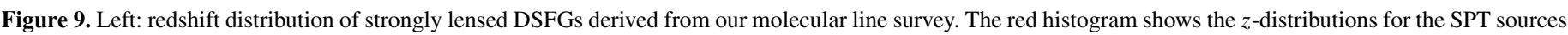

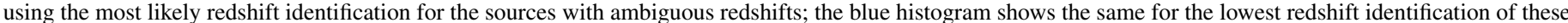

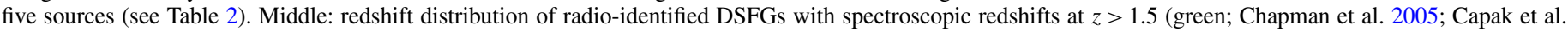

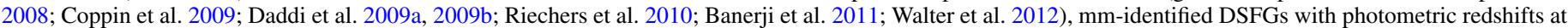

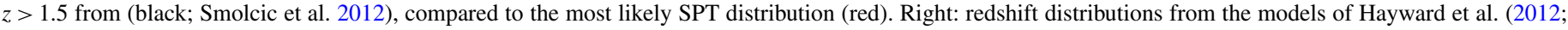

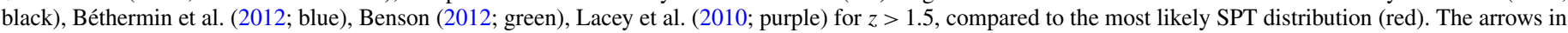

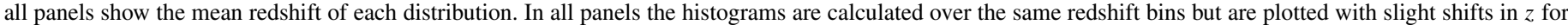
clarity.

These sources, however, were selected to peak in the SPIRE $350 \mu \mathrm{m}$ channel to match the $2.1 \leqslant z \leqslant 3.5$ redshift coverage of the instrument used to measure redshifts (Harris et al. 2012). Despite this selection, $>50 \%$ of their targeted sources remained undetected in $\mathrm{CO}$, which may imply that there are a significant number of sources at redshifts larger than $z=3.5$ in this sample as well.

Smolcic et al. (2012) also find an increased fraction of DSFGs at $z>3$ through a combination of spectroscopic and photometric redshifts for a mixed sample of $1.1 \mathrm{~mm}$ and $870 \mu \mathrm{m}$ selected sources in the COSMOS field. They note that $50 \%-70 \%$ of their $z>3$ DSFGs have no radio counterpart down to $\sim 10 \mu \mathrm{Jy}$ at $1.4 \mathrm{GHz}$, which supports the prediction that including radio counterpart identification in the process of surveying DSFG redshifts will suppress higher- $z$ sources, as expected from SED templates. The similarity in the redshift distribution of unlensed sources compiled by Smolcic et al. (2012), derived primarily from photometric redshifts, and our own (Figure 9, center) may be evidence that gravitational lensing is not strongly affecting the underlying redshift distribution. However, greater numbers of molecular-line-derived redshifts for both populations will likely be required to settle this issue.

In the case of no size evolution in DSFGs, our study suggests that previous spectroscopic DSFG redshift surveys, which are almost exclusively based on radio identified sources, have missed $\geqslant 50 \%$ of the DSFG population as it resides at redshifts $z>3$ and the putative high-redshift tail of DSFGs may in fact turn out to be a much broader, flat-topped redshift distribution which could extend to $z>4$.

\subsection{Comparison to Models}

Redshift distributions $(d n / d z)$ and number counts are the main observational constraints to galaxy formation models. Matching available data for DSFGs with these models has been particularly difficult (e.g., Baugh et al. 2005), requiring some ad hoc changes such as top-heavy initial mass functions. As argued above, our $d n / d z$-although currently based on only 28 sources-appears significantly different from the currently largest sample of spectroscopic DSFG redshifts by Chapman et al. (2005). With direct $\mathrm{mm}$ identifications, a $71 \%$ spectroscopic completeness, and likely redshifts for an additional $18 \%$, our SPT DSFG $d n / d z$ represents an important new observational constraint to these models.
We compare our measured $d n / d z$ with four recent models in Figure 9 (right), removing sources at $z<1.5$ from the models to mimic the strong lensing selection described in Section 4.3. We discuss the individual models below and give the $\chi^{2}$ for each model for the five redshift bins. Despite the relatively small number of redshifts, our new SPT $d n / d z$ already discriminates between galaxy formation models.

Béthermin et al. (2012) present an empirical model starting from the observed FIR number counts split into "main-sequence" and starburst mode star-forming galaxies. Their model includes the effects of magnification by strong lensing, so it can directly predict the $d n / d z$ for the SPT sample. For the comparison with our data we use the predicted redshift distribution for sources with $S_{1.4 \mathrm{~mm}}>15 \mathrm{mJy}$, consistent with our source selection. This model matches our redshift histogram very well, with a comparison to the five redshift bins giving a $\chi^{2}$ of 1.9 across five redshift bins.

The Lacey et al. (2010) model is a semi-analytic model identical to that presented in Baugh et al. (2005). The model employs a top-heavy stellar initial mass function, which results in more luminosity and more dust produced per unit star formation rate, to better match the bright end of $850 \mu \mathrm{m}$ galaxy counts. This model does not include the effects of strong lensing, and DSFG counts are based on a selection in $S_{1.4 \mathrm{~mm}}$ with $>1$ mJy (C. Lacey 2012, private communication). The $\chi^{2}$ between this model and our measurement across the five redshift bins is 10.7 .

The Benson (2012) model is a semi-analytic model that also expands upon the work of Baugh et al. (2005). Whereas the Lacey et al. (2010) model required a top-heavy stellar initial mass function, the Benson (2012) model merely has enhanced dust production in starbursts. This model does not include the effects of strong lensing, and DSFG counts are based on a selection in $S_{850 \mu \mathrm{m}}(>5 \mathrm{mJy})$. The predicted $d n / d z$ distribution comes close to the Chapman et al. (2005) distribution, but clearly fails to fit the SPT or Smolcic et al. (2012) measurements. Part of this difference may be due to the $850 \mu \mathrm{m}$ instead of $1.4 \mathrm{~mm}$ source selection, and a possible lensing bias. The $\chi^{2}$ between this model and our measurement across the five redshift bins is 39.8. Our measurements are clearly at odds with this model.

The model by Hayward et al. (2012) combines a semi-empirical model with 3D hydrodynamical simulations and a 3D dust radiative transfer. Strong lensing is not included in the 
modeling and the model predicted $d n / d z$ is determined using sources with $S_{1 \mathrm{~mm}}>1 \mathrm{mJy}$, consistent with the expected intrinsic flux densities of our sample. The distribution of the DSFGs in this model is close to the observed SPT $d n / d z$, with a $\chi^{2}$ of 2.8 between data and model.

\section{SUMMARY AND CONCLUSION}

We have used ALMA to measure or constrain the redshifts of 26 strongly lensed DSFGs detected in the SPT-SZ survey data. The redshifts were derived using molecular emission lines detected in frequency scans in the $3 \mathrm{~mm}$ transmission window covering 84.2-114.9 GHz. As the molecular emission lines can unambiguously be associated with the thermal dust continuum emission at our selection wavelength of $1.4 \mathrm{~mm}$, this technique does not require any multi-wavelength identification unlike other methods typically used to derive DSFG redshifts.

In total we detect 44 spectral features in our survey which we identify as redshifted emission lines of ${ }^{12} \mathrm{CO},{ }^{13} \mathrm{CO}, \mathrm{C} \mathrm{I}, \mathrm{H}_{2} \mathrm{O}$, and $\mathrm{H}_{2} \mathrm{O}^{+}$. We find 1 or more lines in 23 sources, yielding an unprecedented $\sim 90 \%$ success rate of this survey. In 12 sources we detect multiple lines. In 11 sources we robustly detect a single line, and in 1 of those cases we can use that single line to obtain an unambiguous redshift. For an additional 5 galaxies, in which we detect a single line with ALMA, we can determine the redshift using additional spectral and optical data yielding 18 unambiguous redshifts. For five sources with a single line detection we have used our excellent $\mathrm{mm} /$ submm photometric coverage ( $3 \mathrm{~mm}$ to $250 \mu \mathrm{m}$ ) to narrow the line identification and make a probabilistic estimate for the redshift based on the FIR dust temperature derived from extensive broadband photometric data. In three sources we do not detect a line feature, either because the lines are too weak, or because they are in the redshift desert $z=1.74-2.00$. Adding in two previously reported SPT sources with spectroscopic redshifts from (Greve et al. 2012), we derive a redshift distribution from 28 SPT sources.

We analyze the redshift biases inherent to our source selection and to gravitational galaxy-galaxy lensing. Our selection of bright $1.4 \mathrm{~mm}$ sources imposes a requirement that they be gravitationally lensed, effectively suppressing sources at $z \lesssim$ 1.5 due to the low probability of being lensed at these redshifts. Beyond $z \sim 2$, gravitational lensing does not significantly bias the redshift distribution unless DSFGs undergo a systematic size evolution between $z=2-6$ with decreasing source sizes for higher redshifts. An analysis of the blackbody radii of unlensed DSFGs from the literature does not support the existence of such an evolution, but it also cannot be excluded conclusively at this point.

Our sample mean redshift is $\bar{z}=3.5$. This finding is in contrast to the redshift distribution of radio identified DSFGs which have a significantly lower mean redshift of $\bar{z}=2.3$, and for which only $10 \%-15 \%$ of the population is expected to be at $z>3$ (e.g., Chapman et al. 2005). The redshift distribution of our sample appears almost flat between $z=2-4$. Our study suggests that previous spectroscopic redshift surveys of DSFGs based on radio identified sources are likely biased toward lower redshift and have missed a large fraction $(\geqslant 50 \%)$ of the DSFG population at redshifts $z>3$.

With a $90 \%$ detection rate, our ALMA+SPT CO redshift survey is the most complete DSFG survey to date. It demonstrates the power of ALMA, with its broadband receivers and large collecting area, to provide the critical galaxy redshift information needed to measure the cosmic history of obscured star formation, particularly at the highest redshifts where other techniques falter. The magnification of the SPT sources by intervening mass (factors of $\sim 10$ or more; Hezaveh et al. 2013) has allowed us to obtain these results in the early science phase of ALMA, with only 16 , of the eventual array of $54,12 \mathrm{~m}$ antennas. With the full array, such studies will be possible on unlensed sources, highlighting the enormous scientific impact ALMA will have in the coming decades. With spectroscopic redshifts for a large number of DSFGs, it is now possible to study the conditions of the interstellar medium at high redshift in great detail through spatially resolved spectroscopy of FIR molecular and atomic lines. The SPT sources presented here represent less than $25 \%$ of the entire sample of high-redshift, strongly lensed DSFGs. Obtaining redshifts for the remaining sources will enable us to definitively constrain the redshift evolution of DSFGs.

The authors thank A. Blain and N. Scoville for many useful discussions related to this work, and A. Benson, C. Baugh, C. Hayward, and C. Lacey for providing us with the predicted redshift distributions from their models and useful discussions regarding their implications. This paper makes use of the following ALMA data: ADS/JAO.ALMA\#2011.0.00957.S. ALMA is a partnership of ESO (representing its member states), NSF (USA) and NINS (Japan), together with NRC (Canada) and NSC and ASIAA (Taiwan), in cooperation with the Republic of Chile. The Joint ALMA Observatory is operated by ESO, AUI/NRAO and NAOJ. Based on observations taken with European Southern Observatory Very Large Telescope, Paranal, Chile, with program ID 088.A-0902(C), and with the Atacama Pathfinder Experiment under program IDs 086.A-0793(A), 086.A-1002(A), 087.A-0815(A) and 087.A-0968(A). APEX is a collaboration between the Max-Planck-Institut für Radioastronomie, the European Southern Observatory, and the Onsala Space Observatory. This research has made use of the NASA/IPAC Extragalactic Database (NED) which is operated by the Jet Propulsion Laboratory, California Institute of Technology, under contract with the National Aeronautics and Space Administration. The SPT is supported by the National Science Foundation through grant ANT-0638937, with partial support through PHY1125897, the Kavli Foundation and the Gordon and Betty Moore Foundation. The National Radio Astronomy Observatory is a facility of the National Science Foundation operated under cooperative agreement by Associated Universities, Inc. Partial support for this work was provided by NASA through grant HST-GO12659 from the Space Telescope Science Institute. This work is based in part on observations made with Herschel, a European Space Agency Cornerstone Mission with significant participation by NASA, and supported through an award issued by JPL/Caltech for OT2_jvieira_5. T.R.G. gratefully acknowledges support from a STFC Advanced Fellowship.

\section{APPENDIX A}

\section{SUPPLEMENTARY REDSHIFT INFORMATION}

In this Appendix, we show the supplementary observations that resolve redshift ambiguities in our ALMA observations:

SPT0125-47. The identification of the $98 \mathrm{GHz}$ line as $\mathrm{CO}(3-2)$ is confirmed with a $\mathrm{CO}(1-0)$ detection using the Australia Telescope Compact Array (Figure 10).

SPT0441-46. The identification of the $105 \mathrm{GHz}$ line as $\mathrm{CO}(5-4)$ is confirmed with a [C II] $158 \mu \mathrm{m}$ detection with the First Light APEX Submillimetre Heterodyne receiver (FLASH) on APEX (Figure 11). The low S/N [C I $](1-0)$ detection with ALMA further strengthens this redshift identification. 


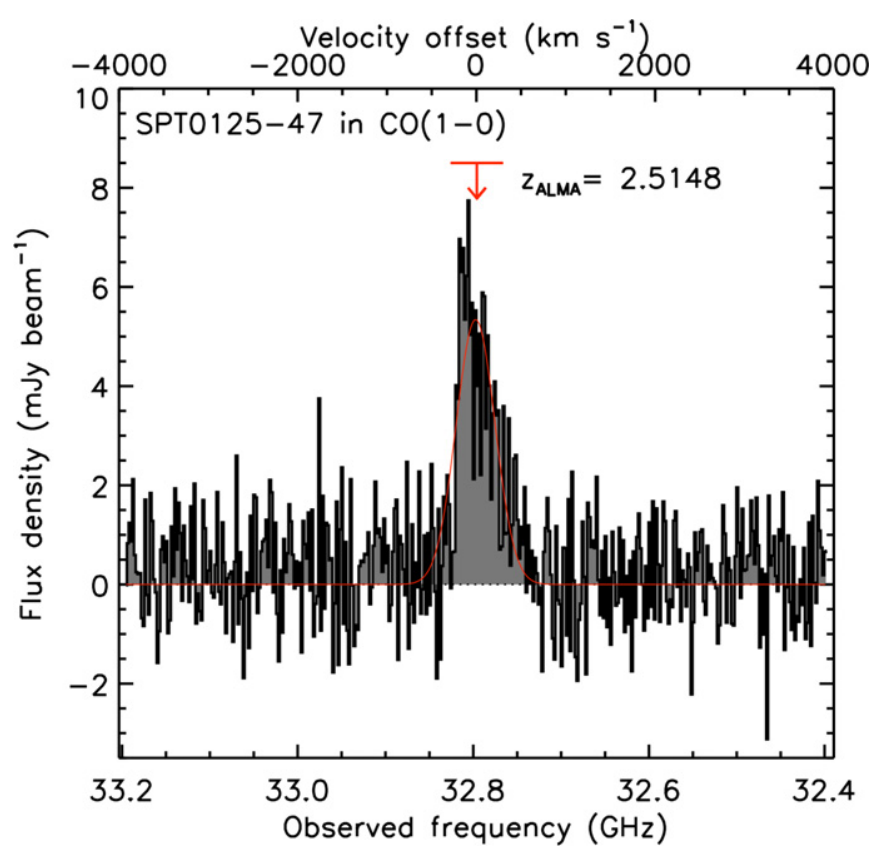

Figure 10. Australia Telescope Compact Array spectrum of SPT 0125-47 showing the $\mathrm{CO}(1-0)$ line confirming the single ALMA line as $\mathrm{CO}(3-2)$ at $z=2.5148$.

(A color version of this figure is available in the online journal.)

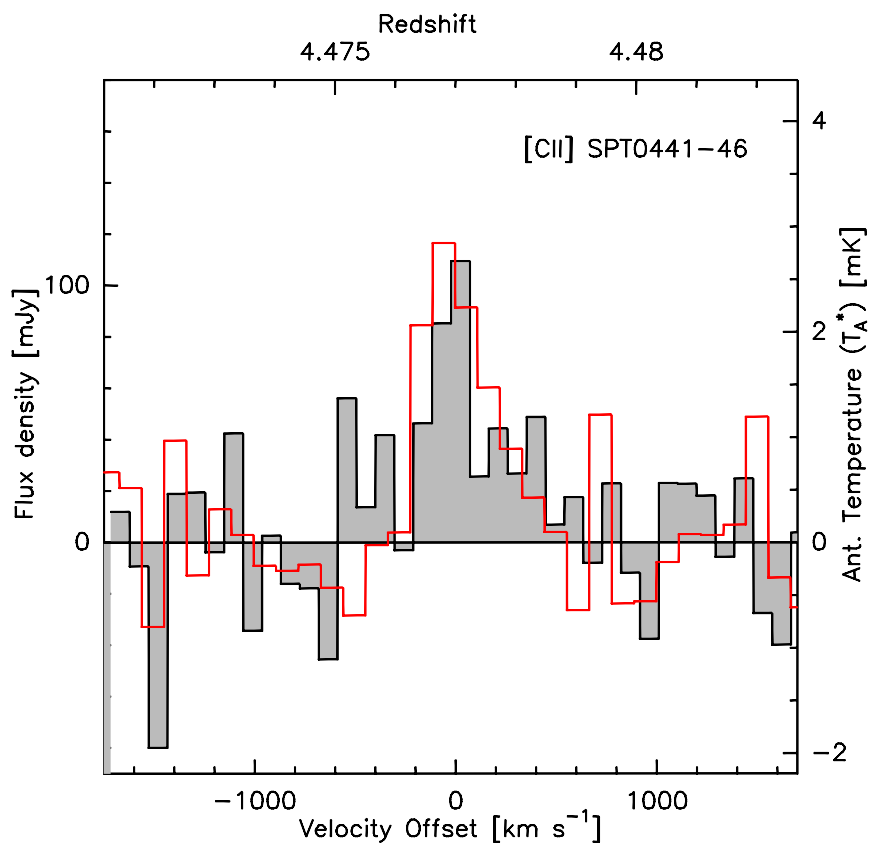

Figure 11. APEX/FLASH spectrum of SPT 0441-46 showing the [C $\mathrm{II}]$ $\lambda 158 \mu \mathrm{m}$ line (filled histogram) confirming the single ALMA line as $\mathrm{CO}(5-4)$ (red line, scaled to allow for a comparison between the line profiles) at $z=$ 4.4771.

(A color version of this figure is available in the online journal.)

SPT0551-50. A strong emission line is visible at $\sim 4800 \AA$ using the VLT FOcal Reducer and Spectrograph (FORS2; Appenzeller et al. 1998), which is consistent with the $3 \mathrm{~mm} \mathrm{CO}(3-2)$ line if we ascribe it to C IV $1550 \AA$. See Figure 12.

SPT2134-50. The $\mathrm{CO}(7-6)$ and $\mathrm{CO}(8-7)$ lines are detected in a 190-310 GHz spectrum (Figure 13) obtained with Z-Spec/APEX (Bradford et al. 2004), and subsequently con-

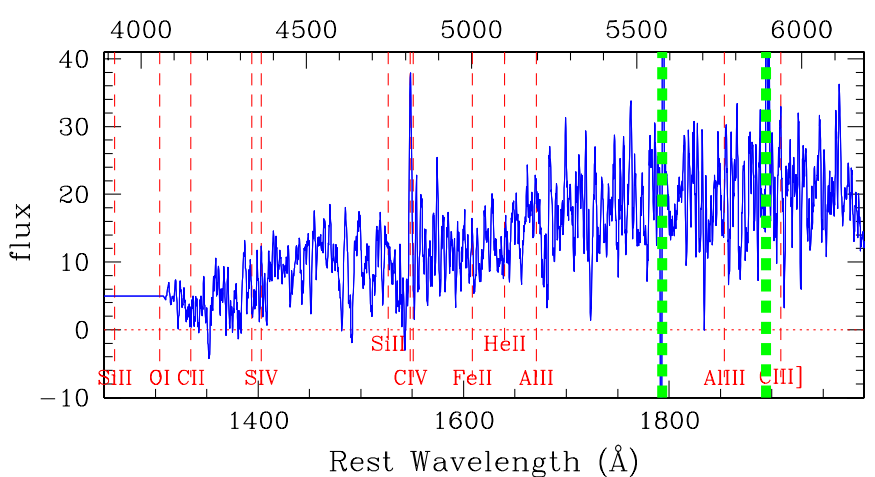

Figure 12. VLT/FORS2 spectrum of SPT 0551-50 showing the C IV $1549 \AA$ line confirming the single ALMA line as $\mathrm{CO}(3-2)$ at $z=2.123$. Thin red dashed lines indicate the wavelengths of expected spectroscopic features, while thick green dotted lines mark areas dominated by skylines.

(A color version of this figure is available in the online journal.)

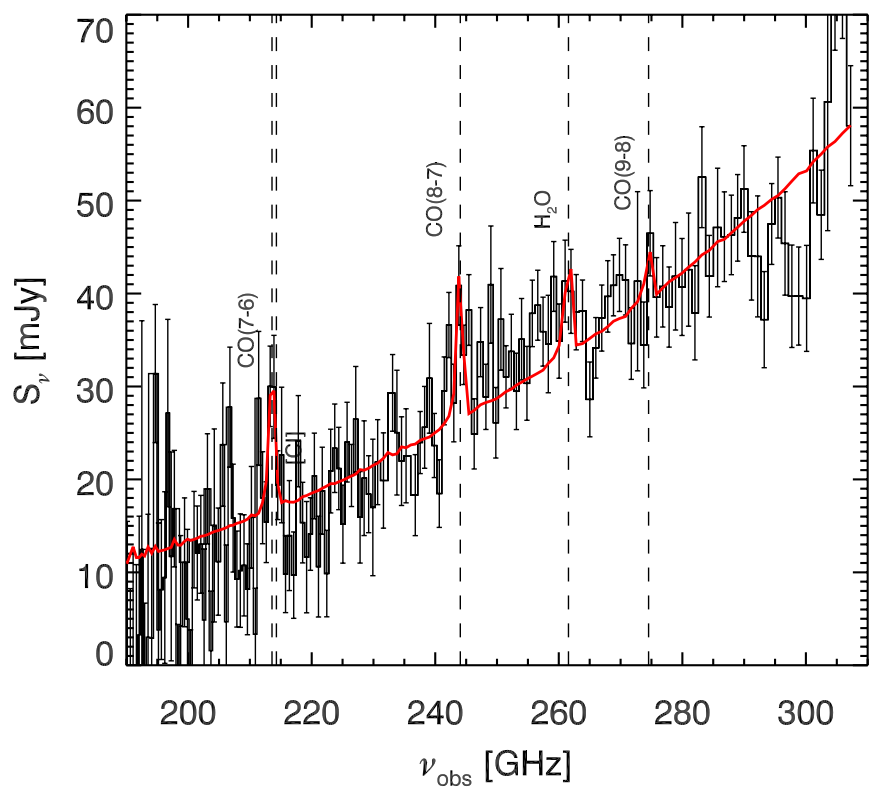

Figure 13. APEX/Z-spec spectrum of SPT 2134-50 showing $2 \sigma-3 \sigma$ detections of the $\mathrm{CO}(7-6)$ and $\mathrm{CO}(8-7)$ lines confirming the single ALMA line as $\mathrm{CO}(3-2)$ at $z=2.779$. Dashed lines mark the expected frequencies of $\mathrm{CO}$ and $\mathrm{H}_{2} \mathrm{O}$ features. The combined significance of the lines detections is 5.6

(A color version of this figure is available in the online journal.)

firmed through Submillimeter Array (SMA) observations of $\mathrm{CO}(7-6)$ and $\left[\mathrm{C}_{\mathrm{I}}\right](2-1)$ (see Figure 14). The ALMA data, released later, agree with this identification, with ALMA detecting the $\mathrm{CO}(3-2)$ line at $91.5 \mathrm{GHz}$.

SPT2132-58. The identification of the $100 \mathrm{GHz}$ line as $\mathrm{CO}(5-4)$ is confirmed with a [C II] $158 \mu$ m detection with the FLASH on APEX (Figure 15).

\section{APPENDIX B}

\section{SUPPLEMENTARY INFORMATION FOR SOURCES WITH A NO OR SINGLE LINE DETECTIONS}

Below, we discuss the nine individual cases which have zero or one CO line detected with ALMA and no additional spectroscopic observations.

SPT0125-50. In this galaxy we detect a second tentative line feature at $99.20 \mathrm{GHz}$ which is consistent with the expected 


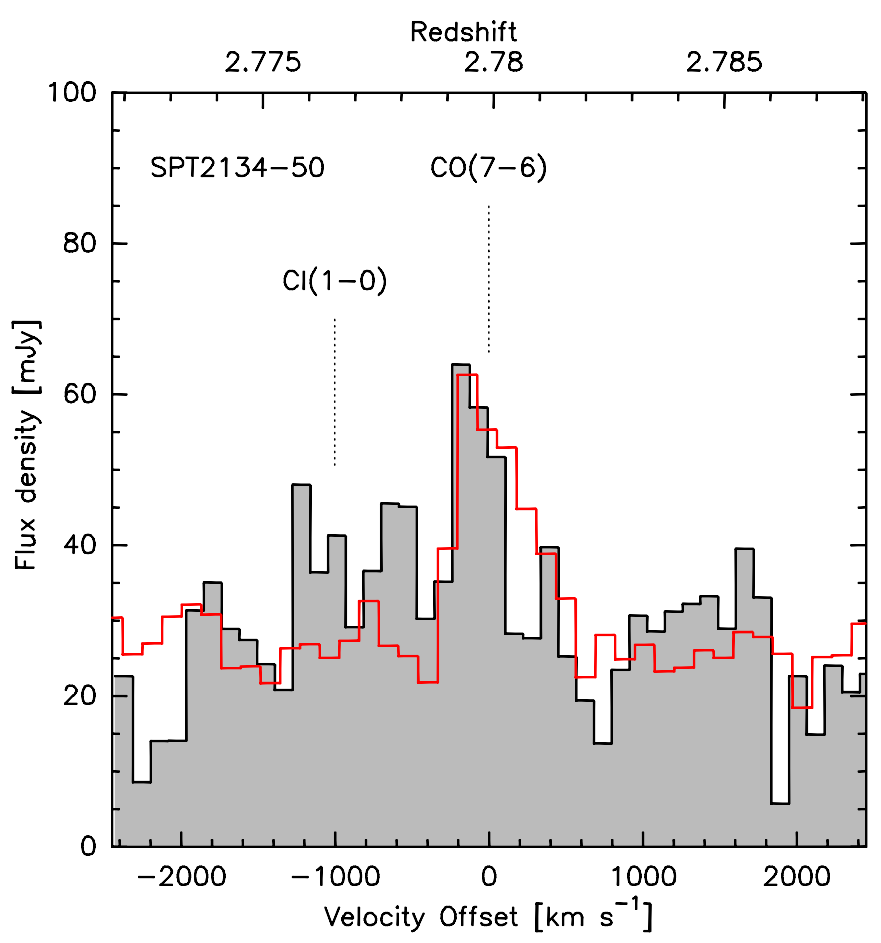

Figure 14. SMA spectrum (filled histogram) of SPT 2134-50 showing CO(7-6) and evidence for $\left[\mathrm{C}_{\mathrm{I}}\right](2-1)$ confirming the single ALMA line as $\mathrm{CO}(3-2)$ (red line, scaled to allow for a comparison between the line profiles) at $z=2.779$.

(A color version of this figure is available in the online journal.)

frequency for $\mathrm{C} \mathrm{I}\left({ }^{3} P_{1} \rightarrow{ }^{3} P_{0}\right)$ if the $93.03 \mathrm{GHz}$ line is $\mathrm{CO}(4-3)$. This is our preferred identification, giving $z=3.959$. In case the weak $99.20 \mathrm{GHz}$ feature is not real, $\mathrm{CO}(5-4)$ as identification for the bright line can be excluded as $\mathrm{CO}(6-5)$ should have been detected too. For $\mathrm{CO}(2-1)$ at $z=1.343$, the implied dust temperature would be $17 \mathrm{~K}$, lower than any we observe. An additional plausible identification is $\mathrm{CO}(3-2)$ at $z=2.717$ $\left(T_{\text {dust }}=30 \mathrm{~K}\right)$.

SPT0128-51. No line is detected in this spectrum. If it is in the $z=1.74-2.00$ redshift desert, the dust temperature is a low $T_{\text {dust }} \approx 19 \mathrm{~K}$. Alternatively, at higher redshift the line-tocontinuum ratio should be smaller and could go undetected. If SPT0128-51 has the same $T_{\text {dust }}$ as the median temperature of the unambiguously identified population, $37 \mathrm{~K}$, its corresponding photometric redshift would be $z=4.3$.

SPT0300-46. This source is similar to SPT0125-50 and has a clear $\mathrm{CO}$ detection at $100.30 \mathrm{GHz}$ and a tentative $\mathrm{C} \mathrm{I}\left({ }^{3} P_{1} \rightarrow{ }^{3} P_{0}\right)$ line at $107.08 \mathrm{GHz}$ which implies $\mathrm{CO}(4-3)$ at $z=3.594$. If the latter feature is not real, $\mathrm{CO}(3-2)$ at $z=2.446$ and $T_{\text {dust }}=27 \mathrm{~K}$ is an alternative interpretation. $\mathrm{CO}(2-1)$ at $z=1.298$ would imply $T_{\text {dust }}=17 \mathrm{~K}$, which we consider unlikely. $\mathrm{CO}(5-4)$ can be ruled out as $\mathrm{CO}(6-5)$ would have also been detected.

SPT0319-47. No line is detected in this spectrum. The dust temperature would be $\approx 20 \mathrm{~K}$ if the source is in the $z=1.74-2.00$ redshift desert. As with SPT0128-51, a higher redshift with weak lines cannot be ruled out. Matching this source to the median temperature of the known sample yields a photometric redshift of $z=4.0$.

SPT0452-50. There is a clear line detection at the very edge of the band (114.87 GHz). $\mathrm{CO}(4-3)$ and $\mathrm{CO}(5-4)$ can be excluded as a second $\mathrm{CO}$ line would be detected in the band. $\mathrm{CO}(2-1)$ at $z=1.007$ can be excluded as it would imply $T_{\text {dust }}=13 \mathrm{~K}$. This identifies the line as $\mathrm{CO}(3-2)$ at $z=2.010$.

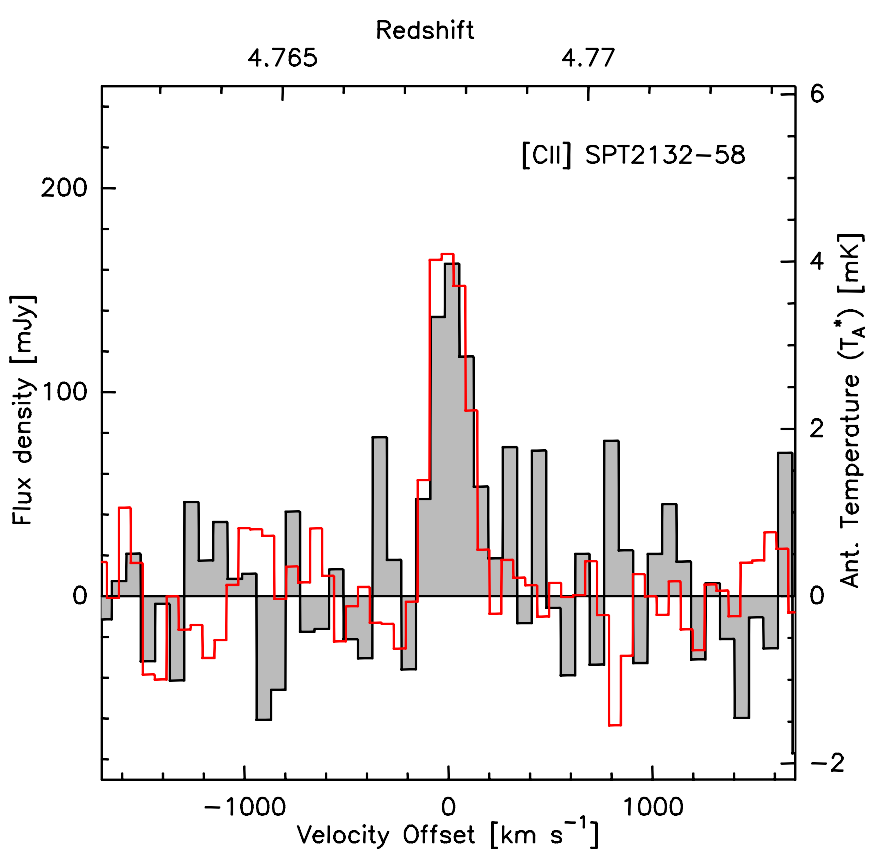

Figure 15. APEX/FLASH spectrum of SPT 2132-58 showing the [CII] $\lambda 158 \mu \mathrm{m}$ line (filled histogram) confirming the single ALMA line as CO(5-4) (red line, scaled to allow for a comparison between the line profiles) at $z=$ 4.7677.

(A color version of this figure is available in the online journal.)

SPT0457-49. There is no line detected in the spectrum. The dust temperature would be $\approx 22 \mathrm{~K}$ if the source is in the $z=$ 1.74-2.00 redshift desert. As with SPT0128-51, a higher redshift with weak lines cannot be ruled out. This source would lie at $z=3.3$ were its $T_{\text {dust }}$ the same as the median of the unambiguous sample.

SPT0459-58. A single CO line is detected at $98.40 \mathrm{GHz}$. If the line is identified as $\mathrm{CO}(4-3)$ at $z=3.685$, the $\mathrm{C}\left({ }^{3} P_{1} \rightarrow{ }^{3} P_{0}\right)$ transition is in the band as well at $105.12 \mathrm{GHz}$. In this case the $\mathrm{C} \mathrm{I}\left({ }^{3} P_{1} \rightarrow{ }^{3} P_{0}\right) / \mathrm{CO}(4-3)$ flux density ratio limit is $<0.15(3 \sigma)$, comparable to the limit we observe for SPT0345-47. Therefore $\mathrm{CO}(4-3)$ cannot be excluded but would require an unusually low (but not unprecedented) $\mathrm{C}$ I/CO line ratio. $\mathrm{CO}(2-1)$ at $z=$ 1.343 can be excluded based on the dust temperature $\left(T_{\text {dust }}=\right.$ $14 \mathrm{~K}) \cdot \operatorname{CO}(3-2)$ at $z=2.514$ implies $T_{\text {dust }}=22 \mathrm{~K}$. The most plausible identification is $\mathrm{CO}(5-4)$ at $z=4.856$ with $T_{\text {dust }}=41 \mathrm{~K}$

SPT0512-59. A single CO line is detected at $106.94 \mathrm{GHz}$. $\mathrm{CO}(4-3)$ and $\mathrm{CO}(5-4)$ can be excluded as $\mathrm{C} \mathrm{I}\left({ }^{3} P_{1} \rightarrow{ }^{3} P_{0}\right)$ should have been detected given the bright $\mathrm{CO}$ line. $\mathrm{CO}(2-1)$ at $z=$ 1.156 is unlikely as it implies $T_{\text {dust }}=20 \mathrm{~K}$, but cannot be ruled out. Our preferred identification is $\mathrm{CO}(3-2)$ at $z=2.234$ with $T_{\text {dust }}=33 \mathrm{~K}$.

SPT0550-53. A single bright $\mathrm{CO}$ line is identified at $111.67 \mathrm{GHz}$. $\mathrm{CO}(2-1)$ at $z=1.064$ is excluded $\left(T_{\text {dust }}=14 \mathrm{~K}\right)$; for $\mathrm{CO}(5-4)$ at $z=4.160 \mathrm{CO}(4-3)$ should have been detected. $\mathrm{CO}(3-2)$ at $z=2.096$ and $\mathrm{CO}(4-3)$ at $z=3.128$ are both plausible identifications with $T_{\text {dust }}=22$ and $31 \mathrm{~K}$, respectively.

\section{APPENDIX C}

\section{SUPPLEMENTARY FAR-INFRARED PHOTOMETRY}

In this Appendix, we show the supplementary FIR through $\mathrm{mm}$ photometric measurements (Table 4) used to determine dust temperatures and assign probabilistic redshift estimates to 

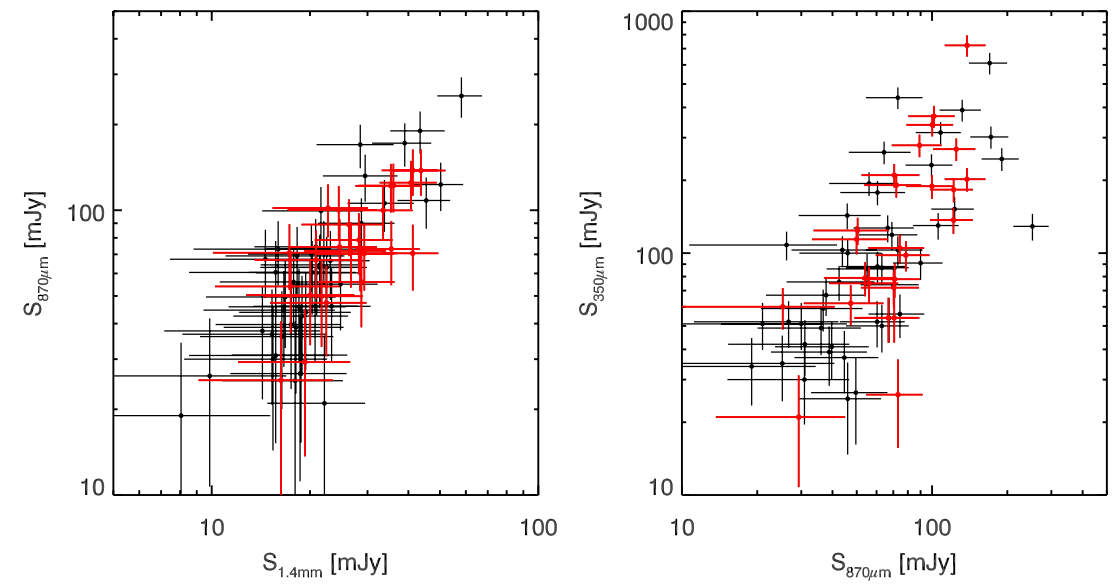

Figure 16. Left: $870 \mu \mathrm{m}$ LABOCA flux density as a function of $1.4 \mathrm{~mm}$ SPT flux density for the 26 sources discussed in this paper (red) compared to the full sample of SPT sources which have been observed with LABOCA and Herschel-SPIRE (black). Right: same as to the left but with $350 \mu \mathrm{m} \mathrm{Herschel-SPIRE} \mathrm{flux} \mathrm{density} \mathrm{as} \mathrm{a}$ function of $870 \mu \mathrm{m}$ LABOCA flux density.

Table 4

Far-infrared and mm Photometry

\begin{tabular}{|c|c|c|c|c|c|c|c|}
\hline ID & $\begin{array}{c}\text { SPIRE } \\
250 \mu \mathrm{m} \\
S_{v}(\mathrm{mJy})\end{array}$ & $\begin{array}{c}\text { SPIRE } \\
350 \mu \mathrm{m} \\
S_{v}(\mathrm{mJy})\end{array}$ & $\begin{array}{c}\text { SPIRE } \\
500 \mu \mathrm{m} \\
S_{v}(\mathrm{mJy})\end{array}$ & $\begin{array}{c}\text { LABOCA } \\
870 \mu \mathrm{m} \\
S_{v}(\mathrm{mJy})\end{array}$ & $\begin{array}{c}\text { SPT } \\
1.4 \mathrm{~mm} \\
S_{v}(\mathrm{mJy})\end{array}$ & $\begin{array}{c}\text { SPT } \\
2.0 \mathrm{~mm} \\
S_{v}(\mathrm{mJy})\end{array}$ & $\begin{array}{c}\text { ALMA } \\
3.0 \mathrm{~mm} \\
S_{v}(\mathrm{mJy})\end{array}$ \\
\hline SPT0103-45 & $121 \pm 15$ & $210 \pm 23$ & $222 \pm 24$ & $132 \pm 22$ & $36.4 \pm 6.8$ & $8.4 \pm 1.6$ & $1.46 \pm 0.23$ \\
\hline SPT0125-47 & $785 \pm 79$ & $722 \pm 73$ & $488 \pm 50$ & $138 \pm 24$ & $41.3 \pm 7.0$ & $8.9 \pm 1.6$ & $1.88 \pm 0.29$ \\
\hline SPT0125-50 & $156 \pm 18$ & $183 \pm 20$ & $156 \pm 18$ & $122 \pm 23$ & $36.0 \pm 6.7$ & $8.1 \pm 1.6$ & $1.51 \pm 0.24$ \\
\hline SPT0128-51 & $40 \pm 9$ & $38 \pm 9$ & $38 \pm 9$ & $29 \pm 8$ & $19.3 \pm 5.5$ & $4.3 \pm 1.5$ & $0.41 \pm 0.09$ \\
\hline SPT0319-47 & $71 \pm 11$ & $105 \pm 13$ & $102 \pm 13$ & $74 \pm 14$ & $24.6 \pm 5.8$ & $5.6 \pm 1.5$ & $1.20 \pm 0.20$ \\
\hline SPT0345-47 & $242 \pm 25$ & $279 \pm 29$ & $215 \pm 23$ & $89 \pm 16$ & $26.3 \pm 6.0$ & $5.3 \pm 1.3$ & $1.48 \pm 0.24$ \\
\hline SPT0346-52 & $136 \pm 16$ & $202 \pm 22$ & $194 \pm 21$ & $138 \pm 24$ & $43.7 \pm 7.1$ & $11.2 \pm 1.6$ & $2.82 \pm 0.43$ \\
\hline SPT0418-47 & $115 \pm 14$ & $189 \pm 20$ & $187 \pm 20$ & $100 \pm 20$ & $33.5 \pm 6.4$ & $7.2 \pm 1.5$ & $0.79 \pm 0.13$ \\
\hline SPT0441-46 & $62 \pm 10$ & $98 \pm 12$ & $105 \pm 13$ & $79 \pm 17$ & $28.2 \pm 6.2$ & $6.8 \pm 1.5$ & $1.26 \pm 0.20$ \\
\hline SPT0452-50 & $38 \pm 9$ & $79 \pm 11$ & $84 \pm 12$ & $54 \pm 10$ & $17.5 \pm 5.2$ & $4.0 \pm 0.9$ & $0.67 \pm 0.11$ \\
\hline SPT0529-54 & $74 \pm 13$ & $137 \pm 17$ & $162 \pm 19$ & $122 \pm 20$ & $35.4 \pm 5.9$ & $9.2 \pm 1.6$ & $1.51 \pm 0.23$ \\
\hline SPT0532-50 & $214 \pm 23$ & $269 \pm 28$ & $256 \pm 27$ & $125 \pm 21$ & $40.8 \pm 6.6$ & $13.4 \pm 1.9$ & $3.04 \pm 0.47$ \\
\hline SPT0550-53 & $65 \pm 18$ & $78 \pm 16$ & $79 \pm 15$ & $71 \pm 15$ & $17.3 \pm 4.6$ & $3.9 \pm 1.1$ & $0.61 \pm 0.12$ \\
\hline SPT0551-50 & $150 \pm 17$ & $191 \pm 21$ & $189 \pm 21$ & $72 \pm 13$ & $26.7 \pm 5.0$ & $5.0 \pm 1.0$ & $1.04 \pm 0.17$ \\
\hline SPT2103-60 & $43 \pm 10$ & $72 \pm 11$ & $108 \pm 15$ & $70 \pm 13$ & $28.5 \pm 5.4$ & $8.1 \pm 1.4$ & $0.99 \pm 0.16$ \\
\hline SPT2132-58 & $55 \pm 11$ & $75 \pm 12$ & $78 \pm 12$ & $56 \pm 10$ & $28.7 \pm 5.5$ & $5.7 \pm 1.2$ & $1.42 \pm 0.23$ \\
\hline SPT2134-50 & $346 \pm 36$ & $339 \pm 35$ & $257 \pm 28$ & $100 \pm 17$ & $24.5 \pm 5.8$ & $5.5 \pm 1.5$ & $1.13 \pm 0.18$ \\
\hline SPT2146-55 & $58 \pm 12$ & $79 \pm 14$ & $82 \pm 14$ & $55 \pm 9$ & $21.8 \pm 5.1$ & $4.7 \pm 1.4$ & $1.18 \pm 0.19$ \\
\hline SPT2147-50 & $73 \pm 12$ & $114 \pm 14$ & $116 \pm 15$ & $50 \pm 9$ & $21.7 \pm 5.2$ & $4.8 \pm 1.5$ & $0.76 \pm 0.12$ \\
\hline
\end{tabular}

Notes. Fluxes are given in units of mJy and include absolute calibration uncertainties. $2 \mathrm{~mm} \& 1.4 \mathrm{~mm}$ fluxes have been deboosted. All other flux densities are photometric measurements at the ALMA position of the $1.4 \mathrm{~mm}$ source. We note that source blending is typically not a problem for the photometry as the strong galaxy-galaxy lensing implies that the FIR light is dominated by a single lensed background object. Contamination by the lensing foreground galaxy can be ruled out by our ALMA high angular resolution $870 \mu \mathrm{m}$ imaging (Vieira et al. 2013).

the sources with single-line detections. Figure 16 shows the representativeness of the dust colors of this subsample for the larger sample of $1.4 \mathrm{~mm}$ selected SPT sources meeting the same selection criteria.

We used the LABOCA instruments at APEX to obtain 870 imaging. The observations took place during ESO and MPIfR observing time between 2010 September and 2012 May. The observing strategy and data processing are described in Greve et al. (2012).
Herschel-SPIRE maps at 250, 350, and $500 \mu \mathrm{m}$ were observed as part of program OT2_jvieira_5. The SPIRE data consists of a triple repetition map, with coverage complete to a radius of 5 arcmin from the nominal SPT position. The maps were produced via the standard reduction pipeline HIPE v9.0, the SPIRE Photometer Interactive Analysis package v1.7, and the calibration product v8.1. Photometry was extracted by fitting a Gaussian profile to the SPIRE counterpart of the SPT detection and the noise was estimated by taking the rms in the 
central 5 arcmin of the map which is then added in quadrature to the absolute calibration uncertainty.

For SED fits, we have added in quadrature an absolute calibration uncertainty of $10 \%$ for SPIRE, $15 \%$ for LABOCA, $10 \%$ for SPT, and $10 \%$ for ALMA.

\section{REFERENCES}

Appenzeller, I., Fricke, K., Fürtig, W., et al. 1998, Msngr, 94, 1 Ashby, M. L. N., Dye, S., Huang, J.-S., et al. 2006, ApJ, 644, 778 Austermann, J. E., Aretxaga, I., Hughes, D. H., et al. 2009, MNRAS, 393, 1573 Banerji, M., Chapman, S. C., Smail, I., et al. 2011, MNRAS, 418, 1071 Baugh, C. M., Lacey, C. G., Frenk, C. S., et al. 2005, MNRAS, 356, 1191 Benford, D. J., Cox, P., Omont, A., Phillips, T. G., \& McMahon, R. G. 1999, ApJL, 518, L65

Benson, A. J. 2012, NewA, 17, 175

Béthermin, M., Daddi, E., Magdis, G., et al. 2012, ApJL, 757, L23

Biggs, A. D., Ivison, R. J., Ibar, E., et al. 2011, MNRAS, 413, 2314

Blain, A. W., \& Longair, M. S. 1993, MNRAS, 264, 509

Blain, A. W., Smail, I., Ivison, R. J., \& Kneib, J.-P. 1999, MNRAS, 302, 632

Bock, D. C.-J., Turtle, A. J., \& Green, A. J. 1998, AJ, 116, 1886

Borys, C., Chapman, S., Halpern, M., \& Scott, D. 2003, MNRAS, 344, 385

Bouwens, R. J., Illingworth, G. D., Labbe, I., et al. 2011, Natur, 469, 504

Bouwens, R. J., Illingworth, G. D., Oesch, P. A., et al. 2010, ApJL, 709, L133

Bradford, C. M., Ade, P. A. R., Aguirre, J. E., et al. 2004, Proc. SPIE, 5498, 257

Capak, P., Carilli, C. L., Lee, N., et al. 2008, ApJL, 681, L53

Carilli, C. L., Hodge, J., Walter, F., et al. 2011, ApJL, 739, L33

Carlstrom, J. E., Ade, P. A. R., Aird, K. A., et al. 2011, PASP, 123, 568

Chapman, S. C., Blain, A. W., Ivison, R. J., \& Smail, I. R. 2003, Natur, 422, 695

Chapman, S. C., Blain, A. W., Smail, I., \& Ivison, R. J. 2005, ApJ, 622, 772

Coppin, K., Chapin, E. L., Mortier, A. M. J., et al. 2006, MNRAS, 372, 1621

Coppin, K. E. K., Smail, I., Alexander, D. M., et al. 2009, MNRAS, 395, 1905

Cox, P., Krips, M., Neri, R., et al. 2011, ApJ, 740, 63

Crawford, T. M., Switzer, E. R., Holzapfel, W. L., et al. 2010, ApJ, 718, 513

Daddi, E., Dannerbauer, H., Krips, M., et al. 2009a, ApJL, 695, L176

Daddi, E., Dannerbauer, H., Stern, D., et al. 2009b, ApJ, 694, 1517

Eales, S., Dunne, L., Clements, D., et al. 2010, PASP, 122, 499

Engel, H., Tacconi, L. J., Davies, R. I., et al. 2010, ApJ, 724, 233

Fathi, K., Gatchell, M., Hatziminaoglou, E., \& Epinat, B. 2012, MNRAS, 423, L112

Frayer, D. T., Harris, A. I., Baker, A. J., et al. 2011, ApJL, 726, L22

Genzel, R., Baker, A. J., Tacconi, L. J., et al. 2003, ApJ, 584, 633

Greve, T. R., Pope, A., Scott, D., et al. 2008, MNRAS, 389, 1489

Greve, T. R., Vieira, J. D., Weiß, A., et al. 2012, ApJ, 756, 101

Harris, A. I., Baker, A. J., Frayer, D. T., et al. 2012, ApJ, 752, 152

Hayward, C. C., Narayanan, D., Kereš, D., et al. 2012, MNRAS, 424, 951

Hezaveh, Y. D., \& Holder, G. P. 2011, ApJ, 734, 52

Hezaveh, Y. D., Marrone, D. P., Fassnacht, C. D., Spilker, J. S., \& Vieira, J. D. 2013, ApJ, in press (arXiv:1303.2722)

Hezaveh, Y. D., Marrone, D. P., Fassnacht, C. D., et al. 2012, ApJ, 761, 20

Hopkins, A. M., \& Beacom, J. F. 2006, ApJ, 651, 142

Hughes, D. H., Serjeant, S., Dunlop, J., et al. 1998, Natur, 394, 241
Ivison, R. J., Greve, T. R., Dunlop, J. S., et al. 2007, MNRAS, 380, 199 Ivison, R. J., Greve, T. R., Smail, I., et al. 2002, MNRAS, 337, 1 Ivison, R. J., Papadopoulos, P. P., Smail, I., et al. 2011, MNRAS, 412, 1913

Ivison, R. J., Smail, I., Papadopoulos, P. P., et al. 2010, MNRAS, 404, 198

Karim, A., Swinbank, M., Hodge, J., et al. 2013, MNRAS, in press (arXiv:1210.0249)

Komatsu, E., Smith, K. M., Dunkley, J., et al. 2011, ApJS, 192, 18

Lacey, C. G., Baugh, C. M., Frenk, C. S., et al. 2010, MNRAS, 405, 2

Lilly, S. J., Le Fevre, O., Hammer, F., \& Crampton, D. 1996, ApJL, 460, L1

Lupu, R. E., Scott, K. S., Aguirre, J. E., et al. 2012, ApJ, 757, 135

Madau, P., Ferguson, H. C., Dickinson, M. E., et al. 1996, MNRAS, 283, 1388

McMullin, J. P., Waters, B., Schiebel, D., Young, W., \& Golap, K. 2007, in ASP Conf. Ser. 376, Astronomical Data Analysis Software and Systems XVI, ed. R. A. Shaw, F. Hill, \& D. J. Bell (San Francisco, CA: ASP), 127

Moshir, M., Kopman, G., \& Conrow, T. A. O. (ed.) 1992, IRAS Faint Source Survey, Explanatory Supplement Version 2 (Pasadena: Infrared Processing and Analysis Center, California Institute of Technology)

Negrello, M., Hopwood, R., De Zotti, G., et al. 2010, Sci, 330, 800

Oliver, S. J., Wang, L., Smith, A. J., et al. 2010, A\&A, 518, L21

Petry, D., \& CASA Development Team 2012, in ASP Conf. Ser. 461, Astronomical Data Analysis Software and Systems XXI, ed. P. Ballester, D. Egret, \& N. P. F. Lorente (San Francisco, CA: ASP), 849

Pope, A., Borys, C., Scott, D., et al. 2005, MNRAS, 358, 149

Pope, A., Scott, D., Dickinson, M., et al. 2006, MNRAS, 370, 1185

Riechers, D. A., Capak, P. L., Carilli, C. L., et al. 2010, ApJL, 720, L131

Riechers, D. A., Cooray, A., Omont, A., et al. 2011a, ApJL, 733, L12

Riechers, D. A., Hodge, J., Walter, F., Carilli, C. L., \& Bertoldi, F. 2011b, ApJL, 739, L31

Rujopakarn, W., Rieke, G. H., Eisenstein, D. J., \& Juneau, S. 2011, ApJ, 726,93

Smail, I., Ivison, R. J., \& Blain, A. W. 1997, ApJL, 490, L5

Smolcic, V., Aravena, M., Navarrete, F., et al. 2012, A\&A, 548, 4

Stern, D., Chary, R.-R., Eisenhardt, P. R. M., \& Moustakas, L. A. 2006, AJ, 132,1405

Story, K. T., Reichardt, C. L., Hou, Z., et al. 2012, ApJ, submitted (arXiv:1210.7231)

Swinbank, A. M., Smail, I., Longmore, S., et al. 2010, Natur, 464, 733

Tacconi, L. J., Genzel, R., Smail, I., et al. 2008, ApJ, 680, 246

Tacconi, L. J., Neri, R., Chapman, S. C., et al. 2006, ApJ, 640, 228

van der Werf, P. P., Berciano, A. A., Spaans, M., et al. 2011, ApJL, 741, L38

van der Werf, P. P., Isaak, K. G., Meijerink, R., et al. 2010, A\&A, 518, L42

Vernet, J., Dekker, H., D’Odorico, S., et al. 2011, A\&A, 536, A105

Vieira, J. D., Crawford, T. M., Switzer, E. R., et al. 2010, ApJ, 719, 763

Vieira, J. D., Marrone, D. P., Fassnacht, C. D., et al. 2013, Natur, in press (arXiv:1305.2723)

Walter, F., Carilli, C., Bertoldi, F., et al. 2004, ApJL, 615, L17

Walter, F., Decarli, R., Carilli, C., et al. 2012, Natur, 486, 233

Walter, F., Riechers, D., Cox, P., et al. 2009, Natur, 457, 699

Wardlow, J. L., Smail, I., Coppin, K. E. K., et al. 2011, MNRAS, 415, 1479

Weiß, A., Downes, D., Neri, R., et al. 2007, A\&A, 467, 955

Weiß, A., Ivison, R. J., Downes, D., et al. 2009a, ApJL, 705, L45

Weiß, A., Kovács, A., Coppin, K., et al. 2009b, ApJ, 707, 1201

Williamson, R., Benson, B. A., High, F. W., et al. 2011, ApJ, 738, 139

Younger, J. D., Fazio, G. G., Ashby, M. L. N., et al. 2010, MNRAS, 407, 1268

Younger, J. D., Fazio, G. G., Huang, J.-S., et al. 2007, ApJ, 671, 1531

Yun, M. S., Scott, K. S., Guo, Y., et al. 2012, MNRAS, 420, 957 